\title{
Review of Epidemiologic Studies on Occupational Factors and Lower Extremity Musculoskeletal and Vascular Disorders and Symptoms
}

\author{
Jennifer C. D'Souza, ${ }^{1}$ Alfred Franzblau, ${ }^{1,4,5}$ and Robert A. Werner ${ }^{1,2,3,4}$
}

The epidemiologic literature on lower extremity musculoskeletal disorders (MSDs), vascular disorders, and occupational mechanical factors is relatively sparse, compared to the low back and upper extremities. The present literature review aims to summarize and evaluate the current literature on lower extremity vascular disorders, MSDs, and symptoms, and to update previous literature reviews. A search was conducted in PubMed. Articles were included if they had a lower extremity musculoskeletal outcome, and a workplace factor(s). They were evaluated based upon their generalizability, exposure, and outcome assessments, study design, and controlling of confounders. Most of the literature has focused on osteoarthritis of the hip and knee, whereas not much research has been done examining the feet/ankles and lower legs. Overall, better exposure assessment is needed to examine the causal pathway between occupational factors and MSDs.

KEY WORDS: lower extremity; work-related; occupational; epidemiology.

\section{INTRODUCTION}

Relative to the work-related upper extremity musculoskeletal disorders and low back disorders, much less attention has been given to the epidemiology of lower extremity musculoskeletal disorders. In 1983, the American Podiatric Association reported that $83 \%$ of industrial workers had foot or lower leg problems, including discomfort, pain or orthopedic deformities (1). A query of nonfatal occupational injuries and illnesses treated in US emergency departments (EDs) identified approximately 250,000 strains or sprains of the ankle, foot, knee, or leg (2). These accounted for $6.4 \%$ of the total annual estimate of occupational

\footnotetext{
${ }^{1}$ Department of Environmental Health Sciences, The University of Michigan School of Public Health, 109 South Observatory, Ann Arbor, Michigan 48109-2029.

${ }^{2}$ Department of Physical Medicine and Rehabilitation, University of Michigan Medical Center, Ann Arbor, Michigan.

${ }^{3}$ Physical Medicine and Rehabilitation Service, Veterans Affairs Medical Center, Ann Arbor, Michigan.

${ }^{4}$ Center for Ergonomics, Department of Industrial and Operations Engineering, The University of Michigan College of Engineering, Ann Arbor, Michigan.

${ }^{5}$ Correspondence should be directed to Dr. Franzblau, Department of Environmental Health Sciences, The University of Michigan School of Public Health, 109 South Observatory, Ann Arbor, Michigan 48109-2029.
} 
injuries treated in EDs. However, this estimate may not correctly capture the true prevalence of lower extremity musculoskeletal disorders as many are chronic in nature.

More recent population-based studies have reported knee symptoms (such as stiffness, swelling and pain) prevalence of 54\% among males (3). Based on a postal survey of men and women aged $40-80$ years, $28 \%$ of the population reported knee pain (4).

Previous reviews of the literature on work-related lower extremity musculoskeletal disorders and symptoms have focused on hip and/or knee osteoarthritis (5-7) and other knee disorders (8). Reviews of the epidemiologic literature on varicose veins have also examined the contribution of occupation to varicose veins (9-12). To date, no reviews have been performed on work-related musculoskeletal disorders of the feet, ankles, or legs.

The aim of this review is to evaluate the epidemiologic literature regarding work-related lower extremity musculoskeletal and vascular disorders and symptoms, and to update the state of the epidemiologic literature where previous reviews have already been done.

\section{METHODS}

A search was conducted in PubMed using the phrases: "lower extremity disorders" (phrase not found, but words yielded some), "lower extremity musculoskeletal disorders," "lower extremity musculo-skeletal disorders" (phrases not found) and "hip/knee/ankle/foot" with "occupational/occupation/work." A search for specific lower extremity musculoskeletal and vascular disorders was done (e.g. varicose veins, osteoarthritis, etc.) when resulting articles examined specific disorder(s) and their association with workplace factors. The search field was limited to the title and abstracts of articles. Additionally, the search was limited to articles in the English language and between the years 1965-August 2004.

Only epidemiologic studies investigating lower extremity (hip, knees, legs, ankle, and feet) musculoskeletal disorders and symptoms and occupational risk factors were chosen. The "related articles" function was used on articles that were pertinent. The "related articles" function is a "PubMed feature that uses a word-weighted algorithm to compare words from the title, abstract, and MeSH headings to calculate a set of PubMed citations that are closely related to the selected article." Studies were also identified through the references and bibliographies of related articles.

Laboratory studies were excluded from this review as well as epidemiologic studies of the low back. However, laboratory studies are cited to provide additional evidence for the causal relationship found in epidemiologic associations and to provide insight into other risk factors that may have been overlooked in the epidemiologic literature.

The studies were primarily evaluated on the strength of the study design and statistical analysis, type of population, outcome, and exposure assessments (e.g. job title, self-report of occupational physical activities), controlling of confounders and cofactors (e.g. BMI, age), and other potential modifying factors (e.g. footwear). An overall score was assigned to each article based on these criteria (13). The score is based on a 5-point scale. In this literature review the following scores are defined as: 1 is very poor; 2 is poor; 3 is fair; 4 is strong; 5 is very strong (note the definitions were not from Kristensen et al. (13)). Studies that had particularly strong study designs (cohort or case-control, prospective), and exposure and outcome assessments were described in further detail. In certain cases (e.g. feet/ankles), few studies were done and all studies were described. In areas where reviews of the epidemiologic literature have already been written, this present review will attempt 
to update the review. Some of the primary source articles, that have already been reviewed, are further described due to their especially strong qualities. The studies are organized by anatomic outcomes (feet/ankles, lower legs, knee, hip/thighs). Thus, studies that examined multiple anatomic outcomes may be mentioned more than once. Finally, recommendations are suggested for future research.

\section{FEET/ANKLES}

Nine epidemiologic studies focused on MS outcomes in the foot and/or ankle. Although these studies were not the strongest from a methodologic standpoint, they are presented due to the limited data available in this area. (Table I).

Riddle et al. conducted a matched case-control study of plantar fasciitis (14). The cases $(n=50)$ were drawn from two outpatient physical therapy clinics and were referred by physicians. Controls $(n=100)$ were also drawn from identical physical therapy clinics as well as from a local church congregation. The cases and controls were matched ( 2 controls: 1 case) on age and gender. Plantar fasciitis was physician diagnosed. A goniometer was used to measure ankle dorsiflexion. Height, weight, regular jogging, and "spending the majority of the workday on their feet" were assessed through questionnaire. The researchers did not differentiate between the times a subject was standing versus walking. The researchers found significant associations between plantar fasciitis and BMI, limited ankle dorsiflexion of the involved side (less than 7.5 degrees) and spending the majority of the workday on feet. The associations were adjusted for "jogging status," age, and gender through multiple logistic regression modeling. Although there was a significant association between plantar fasciitis and spending the majority of the workday on feet (OR: 3.6; 95\% Confidence Interval (CI) 1.3, 10.1), the time spent on feet was a dichotomous variable, was self-reported, and did not separate the possibly different effects of standing and walking. No further data was collected on the extent and duration of the exposure, nor on the particular occupations and work histories of the cases and controls. Furthermore, data on the subjects' footwear, walking/standing surfaces were not available. Also, the study population was drawn primarily from a clinic, which may introduce selection bias. Namely, it is unknown how well the clinic captured all cases of plantar fasciitis, especially those that are work-related. For example, those that were workers' compensation cases and those that were not.

Ryan performed a cross-sectional study of musculoskeletal symptoms in supermarket workers $(n=513)(15)$. An activity analysis was performed on jobs to characterize job titles. Jobs were observed for $30 \mathrm{~min}$ at 10 -s intervals, the subject's posture and activity were recorded. The observations at each of the departments at the store were accumulated to create an "overall activity profile" for each department. The checkout department had the highest observed frequency of workers who were standing. They also had the highest prevalence of ankle/foot and lower limb complaints. The percent of time standing was associated with the number of symptoms reported in the ankle/foot $\left(R^{2}=0.951, p=0.001\right)$. The checkout department was overwhelmingly female $(92.2 \%)$ and was quite young $(60 \%$ of the workers were under 20 years old), making the results of the study difficult to generalize to the overall working population. The analysis did not adjust for BMI, work history, and possibly other important confounders. However, the occupational exposure assessment was a major strength. 


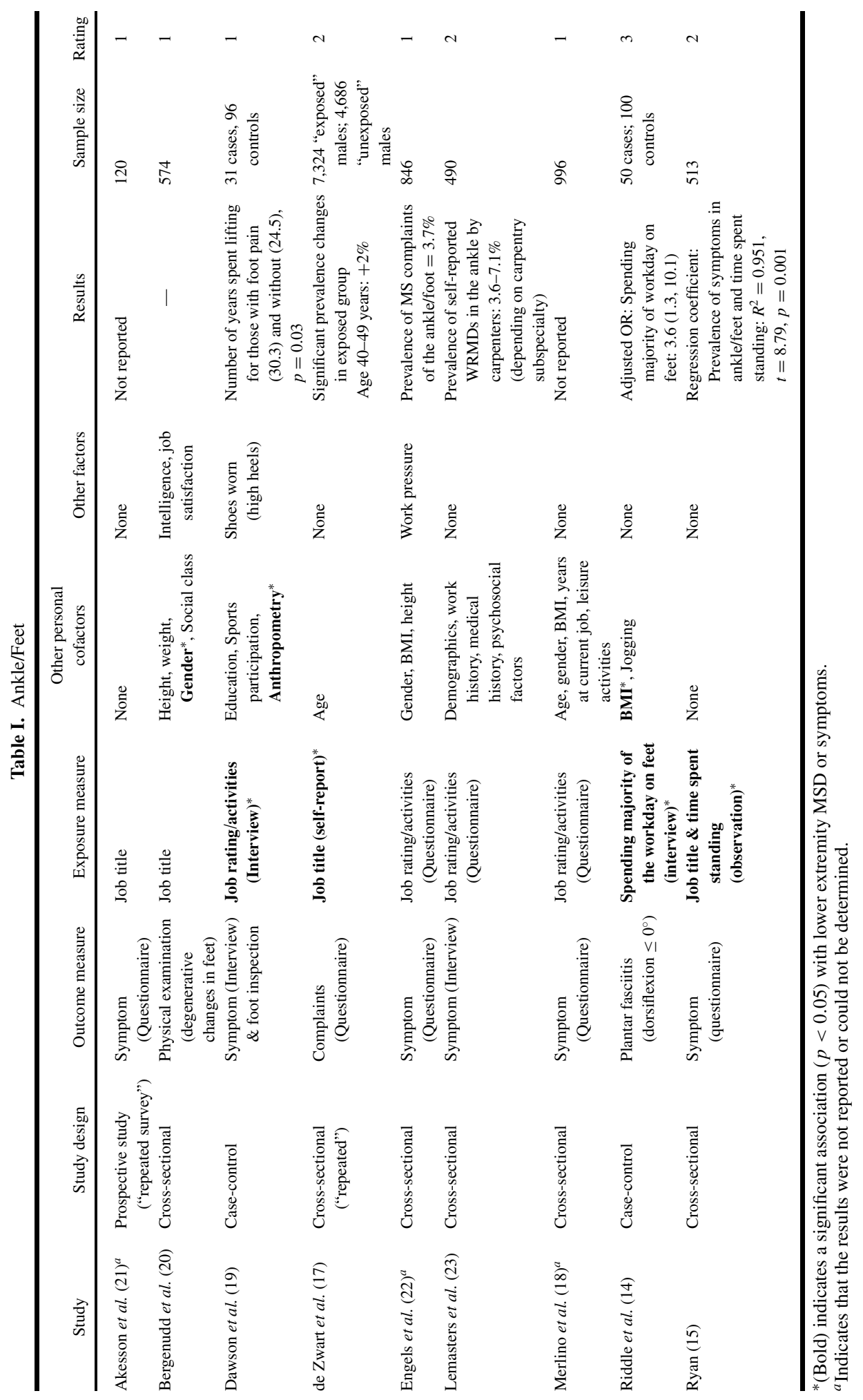


deZwart et al. examined changes in musculoskeletal complaints over 4 years in a Dutch, male working population, with respect to age and work demands (16). Regional occupational health services are responsible for employees of affiliated companies. Work demands were dichotomized into "heavy physical" and "mental work" through job titles. Heavy physical demands aimed to include long periods of standing, awkward postures, stooping, and strenuous work. The work demands of each job title were validated by questionnaire data of employees (17). Musculoskeletal complaints were assessed through surveys. The survey was administered to an age-stratified sample of male employees. The researchers found significant increase of $2 \%$ in prevalence of musculoskeletal complaints in men who had heavy physical demands, in the ankle and feet/toes, but only in the 40-49-year age strata. Although the study population was extremely large (total $n=12,010$ ) and examined changes over time, the poor exposure information and lack of data on additional variables (apart from age), limit the value of the results.

Merlino et al. examined the prevalence of musculoskeletal symptoms (MS) in young construction workers $(n=996)(18)$. Demographic information was collected. A modified version of the Standardized Nordic Questionnaire was used to assess musculoskeletal symptoms. Job factors were self-reported on a scale of 0-10. Also subjects were asked about their participation in various leisure activities. Approximately $9.5 \%$ of the subjects who reported musculoskeletal symptoms in the feet missed work due to the symptoms. In a 12-month period, $23.2 \%$ of the subjects reported musculoskeletal symptoms in the feet, and $3.0 \%$ of the subjects saw a physician for these symptoms. The prevalence of MS symptoms in the feet did not appear to be significantly associated with gender. However the researchers chose to focus on symptoms of other anatomic sites and not on the feet. Thus no associations between feet symptoms and work activities were reported. Also, there was no data presented on standing and walking. Other variables that were not accounted for were psychosocial variables and work history. Although work history was requested of the subjects, it was not reported.

Dawson et al. conducted a population-based study of older (aged 50-70 years) women (31 cases, 96 controls) (19). There was a high prevalence of foot problems (83\%), including foot pain, corns, and bunions. But only those who reported foot pain had spent significantly "more years that involved regular lifting" (30.3 years vs. 24.5 years, $p=$ 0.03), and not other work activities (e.g. standing, lifting, walking, kneeling, etc.). Although data was collected on the type of shoes worn (specifically high heels), this variable could not be examined statistically as $92 \%$ of the subjects had worn 2-inch heels.

Bergenudd et al. (20) examined degenerative changes in the hands and feet (e.g. hammer toes, pes transverso planus, halux valgus) in 574 men and women, aged 55 years at the time of physical examination. Since the subjects were part of a larger longitudinal study (Malmo Longitudinal Study), data on occupational history already existed. Occupation was classified into three groups, according to physical demands: light (e.g. teachers, office clerks, and housewives), moderate (e.g. nurses, shop assistants, bakers), and heavy (e.g. carpenters, bricklayers). Although examples were given of each occupational physical demand category, it is not clear what specific factors caused an occupation to be classified as more physically demanding than another. Associations with height, weight, gender, social class, and intelligence (based upon a national exam given to subjects when they were third graders) were also examined. The overall prevalence of degenerative changes of the study population was $16 \%(n=92)$ and was more prevalent among women. However, there was 
no difference in the prevalence of physical demanding jobs among those with and without degenerative changes of the feet.

Some studies asked about musculoskeletal symptoms in the feet, but the results were not reported (21). Also, a low prevalence (less than 10\%) of ankle/foot MS complaints was reported in some studies (22). Lemasters et al. asked about musculoskeletal symptoms in the ankle and feet in carpenters, but no significant associations were found (23). Gamperiene et al. also looked at symptoms in the ankle and feet, but all lower extremity complaints and symptoms were combined in analyses (24).

Overall, it appears that high BMI, standing, and high physical workload may be associated with ankle/feet musculoskeletal symptoms and plantar fasciitis. However, these observed associations are not adjusted for floor surfaces and type of shoes, which have shown to affect fatigue and discomfort of the feet in laboratory studies (25-27). Although Dawson et al. examined the effect of shoes, the focus was primarily on high heels and not on the actual shoe materials or everyday-type work shoes (19).

\section{LOWER LEGS}

The epidemiologic literature on the lower legs has primarily emphasized vascular diseases. Many of these studies have large study populations with fairly strong study designs though few of the studies were performed in occupational populations. Leg symptoms were also examined, but more specific outcomes such as leg swelling were not. Additionally, the studies examining leg symptoms were cross-sectional (Table II).

\section{Varicose Veins (VV) and Venous Disease}

Reviews of the epidemiologic literature on varicose veins (VV) have examined the evidence for the association between standing at work and varicose veins (9-12) and venous disease $(11,12)$. Callum concluded that the epidemiologic evidence supports the association between varicose veins and the female gender, older age, parity, and country of residence (9). The finding that prevalence of varicose veins varies by country (i.e. lower prevalences are found in underdeveloped countries) suggests a possible role of "western lifestyle" (i.e. sedentary lifestyle, consuming a diet low in fiber) in the development of varicose veins (10). Callum also suggests that race may also explain differences in prevalence (9). There was not enough evidence for the association between VV and obesity, family history, and occupational factors. Callum also concluded that an association between occupation and VV would be difficult to show statistically (9). Unfortunately, no universally accepted standard definition exists for VV. Additionally, VV's recurrent nature (as observed commonly among pregnant women) complicate outcome assessment. The review by Evans et al. came to similar conclusions regarding the problems of assessing VV (10). The literature reviewed by Evans et al. did not consistently show an association between standing posture and VV (10). Hobson also looked at some French studies that examined VV and venous insufficiency, which did not consistently show an association between standing and venous disease (11). However, Hobson ultimately concluded that evidence exists to support the association between prolonged standing at work and venous disease of the lower limbs. Jawien reviewed the literature on chronic venous insufficiency (CVI) in the general population while relating it to a recent large Polish survey that examined individuals for VV and CVI (12). Jawien 


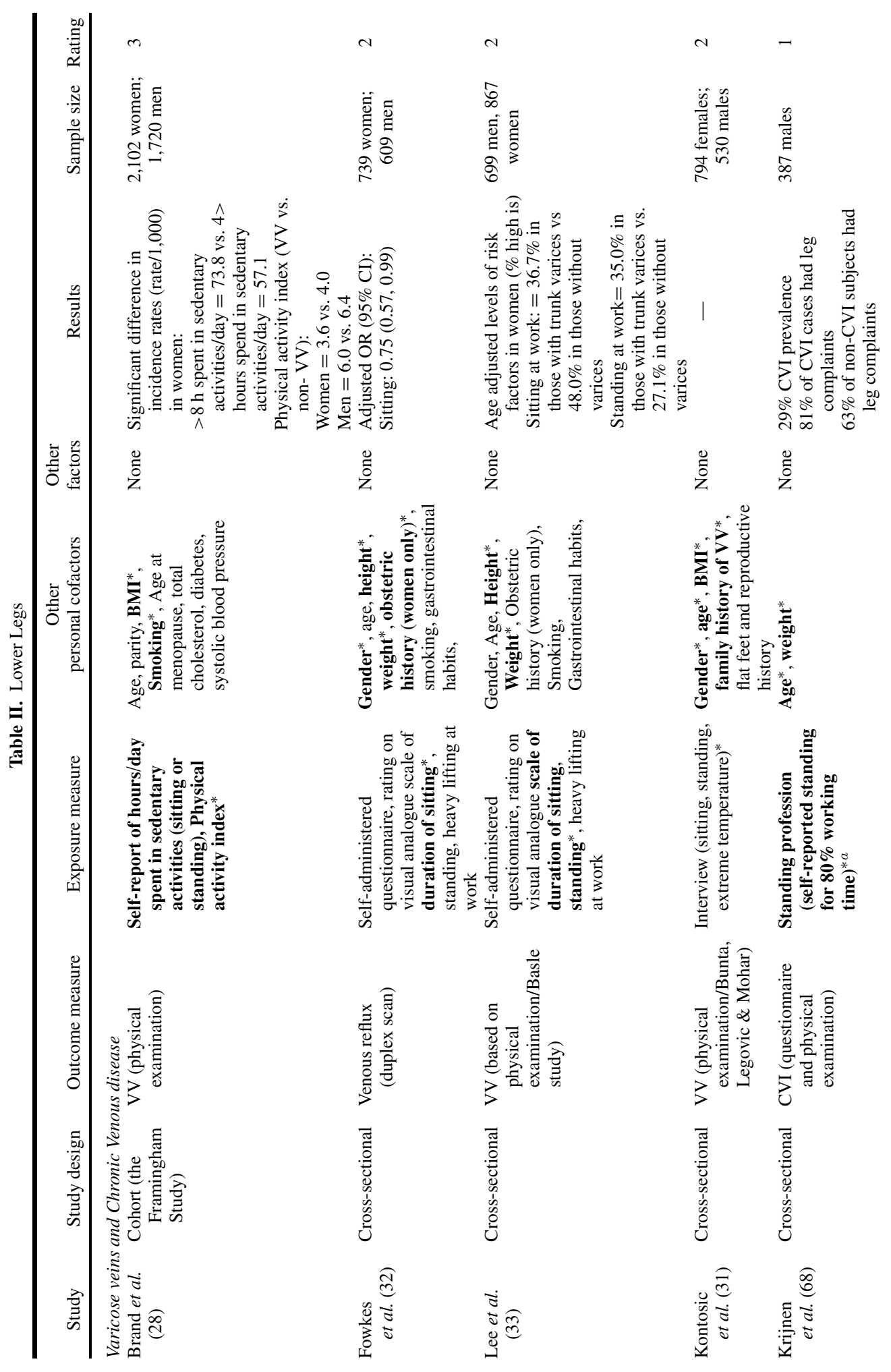




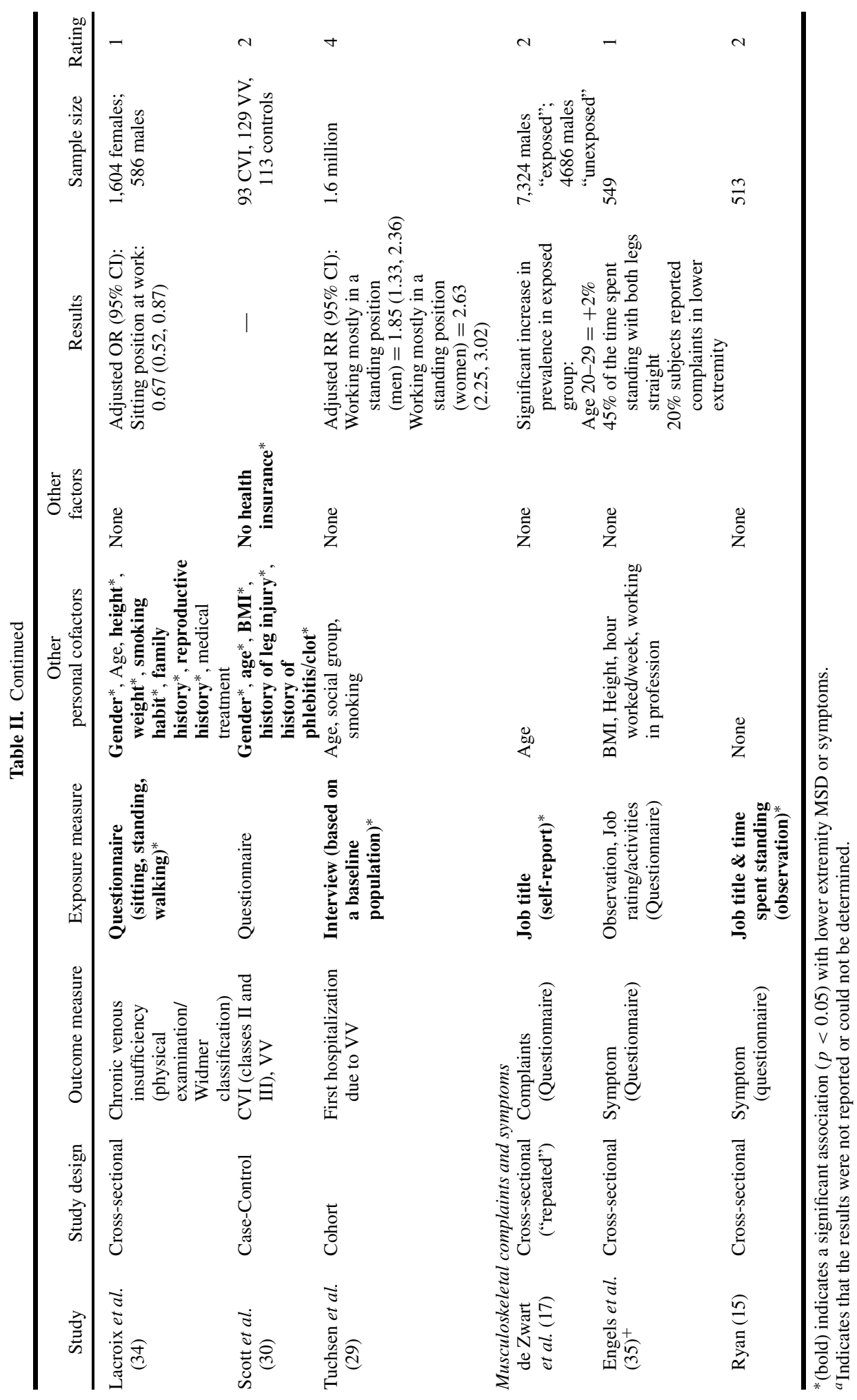


concluded that the contribution of standing and sitting to the development of varicose veins was unclear. The results of the Polish survey did not show a significant association between prolonged sitting (self-reported) and chronic venous disease. However, significantly more subjects who were diagnosed with CVI had occupations that required prolonged standing. It is unclear how these occupational exposures were determined.

Brand et al. examined risk factors for varicose veins (VV) in the large cohort of adults (1720 men and 2102 women) in the Framingham Study (28). Varicose veins (VV) were determined through physical examination and were defined as "the presence of distended and tortuous veins, clearly visible in the lower limbs with the subject standing." The study population was aged 30-62 years, but the percent employed was not reported. Specific occupational exposures were not obtained, but an overall physical activity index was calculated for each subject, based upon self-reported number of hours per day spent in activities of varying intensities. Women who spent greater than $8 \mathrm{~h}$ in sedentary activities had a significantly higher 2 -year age-adjusted incidence ( 73.8 per 1000 ) when compared to women who spent less than $4 \mathrm{~h}$ in sedentary activities (57.1 per 1000); no significant differences in incidence were found in men. However, based on the physical activity index those with VV were significantly less physically active than those who did not have VV. In addition, systolic blood pressure, older age at menopause, and parity were significant risk factors in women (after adjustment for age and other cofactors). BMI was a significant risk factor for VV in women only, while smoking was a significant risk factor for VV in men only. The prospective study and the availability of data on relevant risk factors are major strengths in this design. However, the occupational exposure assessment is problematic. Combining sitting and standing activities does not address the role of posture on the development of varicose veins. The issue of physical activity is more complex as it is closely associated with other risk factors of VV such as BMI.

Tuchsen et al. conducted a large ( $n=1.6$ million) cohort study of 20-59-year-old employed Danes (29). The follow-up period was 3 years. Subjects were obtained through the Occupational Hospitalization Register, which is a research register with individual level data on occupations, hospitalizations, and deaths, as well as follow-up periods. Occupational exposure was assessed through a telephone interview of 8664 adults that were representative of the occupational groups from the Occupational Hospitalization Register. Questions were asked about the physical and psychosocial work environments, work processes, and lifestyle. The data collected was meant to represent the exposures of the study population. VV was assessed through the first hospital admission due to varicose veins (according to ICD-8 code: 454 , varicose veins of the lower extremities - including with ulcer, with stasis dermatitis but without ulcer, other, and unspecified without ulcer). After adjustment for age, smoking, and social group, those who had medium (RR for Men: 1.25; 95\% CI: 1.08, 1.44. RR for Women: 2.97; 95\% CI: 1.75, 2.21) or high proportions (RR for Men: 1.58; 95\% CI: 1.30, 1.91. RR for Women: 2.29; 95\% CI: 2.02, 2.60) of standing work had a significantly higher risk than those who had a low proportion of standing work. However, the researchers recognize that the subject population may be biased as those in more physically demanding occupations, may be more likely to seek hospital treatment (i.e. surgery) than other occupations, or that health seeking behaviors may also be dependent on residence and social class. These biases may lead to an overestimation of the association. Additionally, an overestimation of the association may be likely since patients who seek medical treatment for VV in private clinics are more likely to be of a higher socioeconomic status and are not captured in the register, though this possibility is small. Also, the researchers generalize self-reported 
exposure of a sample of workers to the study population. This may not be accurate if exposures within an occupational category are highly variable. This study's longitudinal design and large cohort are major strengths. It avoids the bias that may occur when disease status influences the recall of work exposures.

Scott et al. (30) conducted a dual case-control study that examined CVI (classes II and III; $n=93)$ and VV $(n=129)$ that were being treated at a local vascular surgery clinic. Control subjects $(n=113)$ were patients from the general surgery clinic and that did not report any history of VV or leg ulcer. Data on relevant risk factors were obtained through survey. The results of the multiple logistic regression analysis did not show any significant association with frequency of standing and VV or CVI. The study had a rather small sample size and did not describe in further detail the method of assessing occupational exposure. Since the analysis also looked at CVI and VV as separate outcomes, the researchers were able to distinguish factors associated with CVI that may differ from the factors associated with VV.

Kontosic et al. conducted a large (males $=530$; females $=794)$ cross-sectional study of workers in Rijeka, Croatia (31). The study population was divided into five exposure groups based upon their type of work (trade, catering, office workers, light industry, and heavy industry). Also, subjects were given a physical examination for VV, and were interviewed to obtain information on workplace exposures, family history of $\mathrm{VV}$, and reproductive history (in women only). Similar to previous studies, a higher prevalence of VV was found in women than in men. Those in the catering and trade groups had a higher prevalence of $\mathrm{VV}$, which were also the groups that were predominantly women. However, standing was only crudely associated with VV and became insignificant after adjustment for age, gender, BMI, and reproductive history.

The Edinburgh Vein Study $(32,33)$ was a large $(n=1566)$ cross-sectional survey of men and women aged 18-64 years randomly selected from computerized age-sex registers of 12 general practices, to capture the Edinburgh, Scotland population. Fowkes et al. examined venous reflux in the lower limbs through duplex scanning (32). Occupational exposures (time spent sitting, standing, walking, and heavy lifting), smoking habits, bowel habit, and obstetric history (for women only) were assessed through self-administered questionnaires. Bowel habits were examined due to the hypothesis that constipation causes increased pressure on the iliac vein, which may lead to varicosities. A significant protective effect was seen between oral contraceptive use, sitting at work, and venous reflux (age-adjusted) in only women. In men, increased height and straining at stool (constipation) were associated with higher odds of venous reflux (age-adjusted), but no occupational exposures were found to be significantly associated. The Edinburgh Vein Study examined VV as an outcome (33). VV was assessed through physical examination and was classified as: varicose veins, chronic venous insufficiency (with skin changes), and chronic venous insufficiency (with healed or active ulceration). But the data resulted in similar finding regarding occupational exposures: insignificant among men and sitting was protective in women while high levels of standing at work was significantly more prevalent in those with varices than those without. However the response rate in the Edinburgh Vein Study was low (54\%) and contrary to most of the previous literature, the study's male population had a greater prevalence of VV than the women.

Another recent cross-sectional study was conducted by Lacroix et al. in an occupational population in Southern Europe (34). The researchers examined 1604 females and 586 males seen at an occupational medicine consultation for routine check-ups. A questionnaire was 
administered to obtain information regarding family history of vein thrombosis and venous insufficiency, sclerotherapy, venous surgery, limb injury, number of pregnancies, smoking habits, and medical treatments. Height and weight were measured. Occupational exposures (sitting, standing, and walking) were assessed through questionnaire as well. Chronic venous insufficiency was determined through the "Widmer classification"-which uses symptoms and observable signs to classify severity of venous insufficiency. The overall prevalence of CVI (all classes) in this population was $51.4 \%$. A multivariate analysis was conducted that included gender, age, family history, BMI, history of leg injury, and a sitting position at work. All the variables were found to be significantly associated with CVI. Standing was not found to be significant, though only $5 \%(n=109)$ of the study population reported that they worked standing.

The recent literature has only provided fair evidence for the association between occupational exposures and VV and CVI. Although sitting at work was found to be protective (32-34), it was mostly found in women. Standing was found to be significantly associated with VV $(28,29)$. But the results from the Framingham study implicate low physical activity in the development of VV. Overall, the relationship between occupational exposures and VV and CVI remains unclear. The measurement of occupational exposure is based on selfreport and the outcome assessments vary widely and do not adequately address the possible roles of posture and physical activity in the development of VV or CVI. Fowkes et al. used duplex scanning to measure venous reflux, which may be a more objective method of assessing VV or CVI (32).

\section{MS Symptoms and Complaints}

de Zwart et al. studied the changes in musculoskeletal complaints in male employees and observed an increase in prevalence of lower leg complaints in the heavy physical demand group, but only in the 20-29-year age stratum (16). But, as aforementioned, the limitations of the study make it difficult to explain why the finding is limited to the particular age stratum.

A cross-sectional study of supermarket workers (15) found an association between the amount of time spent standing and the number of lower leg complaints. Also, departments where the workers spent most of the time standing, had the highest prevalence of lower leg complaints.

Thirteen percent of nursing staff (total $n=549$ ) in nursing homes had MS complaints in knee/lower legs (35). The analysis was restricted to female subjects due to the overwhelming $(80 \%)$ prevalence of women working in Dutch nursing homes. There was no comparison group. The study did use observations to assess exposure.

Aside from the above-mentioned studies, most studies that examined lower leg symptoms and complaints, analyzed the data together with all lower extremity complaints and symptoms. Based on the few studies examining leg symptoms, time spent standing was associated with leg symptoms. de Zwart et al. found a significant association between "heavy physical demand" and leg complaints, but it is difficult to determine which occupational activity is actually relevant to the lower legs (16), since many studies did not specify where in the leg the complaints occurred. Laboratory studies suggest that the leg swelling and discomfort due to prolonged standing is related to the type of flooring $(25,36)$ and workrest scheduling (37). The epidemiologic literature on VV and CVI provides inconsistent 
evidence for an association between occupational factors and CVI and VV. However, specific occupational activities (e.g. standing, sitting, and walking) have not been adequately examined.

\section{KNEE}

Epidemiologic studies on knee OA and occupation were more frequent than other knee disorders or symptoms/pain. Ten studies regarding knee pain and symptoms and four studies regarding knee OA are described. The knee OA studies have stronger study designs than those of knee symptoms/pain (Table III).

\section{Osteoarthritis (OA)}

Schouten et al. (7), Maetzel et al. (5), and Jensen et al. (38) reviewed the epidemiologic literature on occupational exposure and knee osteoarthritis. Jensen et al. concluded that several of the studies had insufficient control of confounding, poor characterization and assessment of exposures and outcome, and small sample sizes (38). Additionally, the reviewers mentioned that the different definitions of knee OA resulted in wide variation of reported prevalences. Despite the shortcomings, the overall epidemiologic literature that was reviewed provided strong evidence for an association between knee OA and kneeling or squatting work and heavy physical work.

The review by Maetzel et al. concluded that the association between knee bending at work and knee OA consistently was positive only in males (5). Similar to the conclusions of Jensen et al., better occupational exposure data is needed (38). Schouten et al. reviewed only literature in 2000 and 2001, and the studies reviewed ( $n=4$ articles) did not consistently show a relationship between kneeling and squatting and knee OA (7).

Lau et al. conducted a matched case-control study with 1 to 1 matching in subjects with osteoarthritis of the knee (39). The cases (166 men, 492 women) and controls (166 men, 492 women) were age and gender matched. Cases were patients in orthopedic units of regional hospitals in Hong Kong. Radiographs of the cases were used to confirm the osteoarthritis. Controls were recruited from general practice clinics, local to the study hospitals. Controls had no self-reported musculoskeletal disorders, pain, or stiffness in the hip/knee and had no "Western practitioner" diagnose them with OA. Data was collected by interview. Questions were asked regarding occupational activities in the subject's "longest held job." In both genders, lifting more than $10 \mathrm{~kg}$ more than 10 times per week was positively associated with knee OA (OR men: 5.4; 95\% CI: 2.4, 12.4; OR women: 2.0; 95\% CI: 1.2, 3.1). Climbing more than 15 flights of stairs per day was only significantly associated with knee OA in women (OR: 5.1; 95\% CI: 2.5, 10.2). Also, height, weight, and history of joint injury were positively associated with osteoarthritis of the knees. However, the use of hospital patients as cases may introduce selection bias as it may not capture all cases or capture cases that are occupationally disadvantaged secondary to osteoarthritis.

Yoshimura et al. (40) performed a case-control study in Japan to investigate risk factor differences between Britain and Japan. Cases $(n=101)$ were obtained from six hospitals and were women older than 45 years, suffering from knee pain and "walking difficulties," and had a Kellgren-Lawrence score of grade 3 or greater. A control $(n=101)$ was randomly 


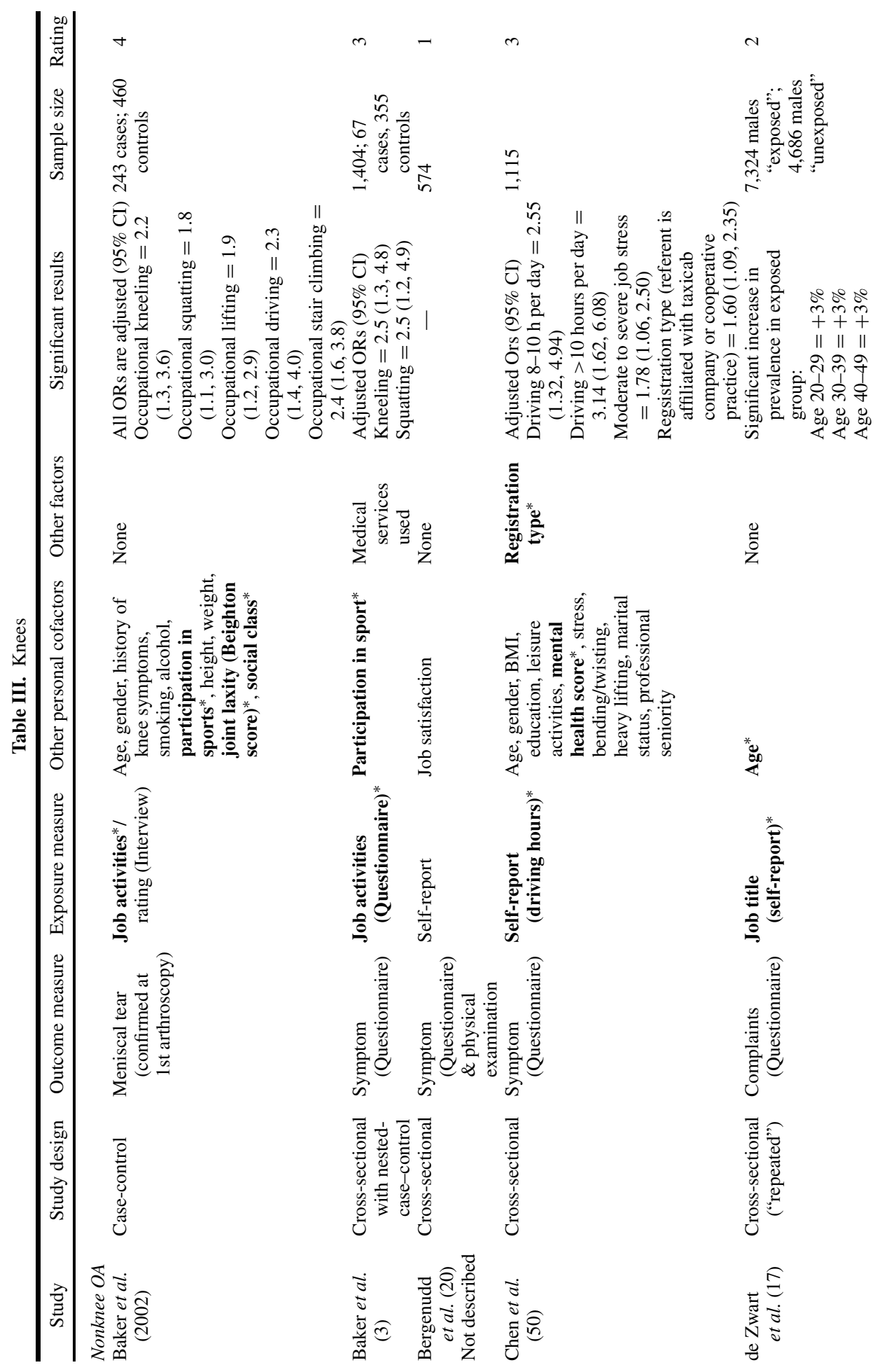




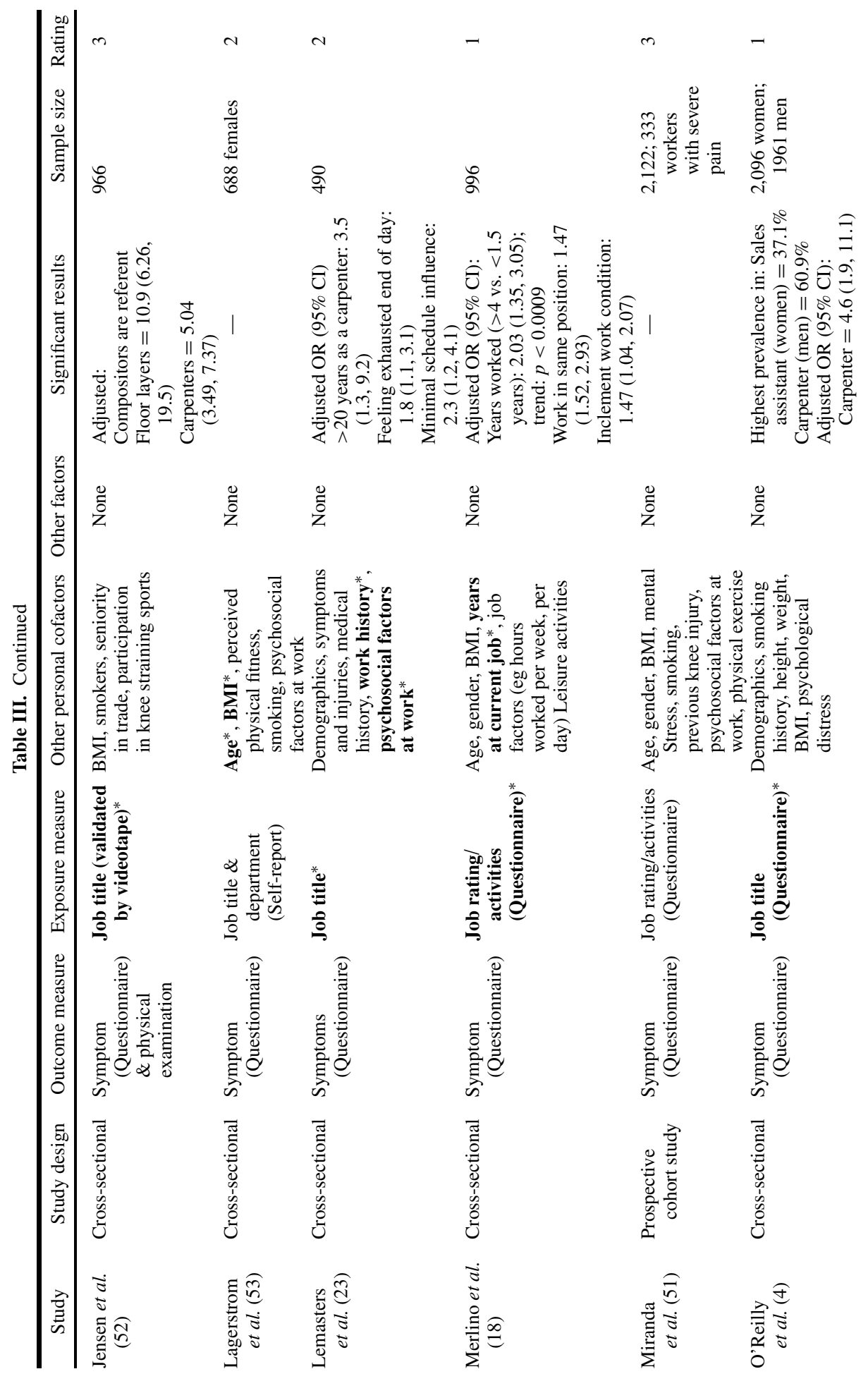




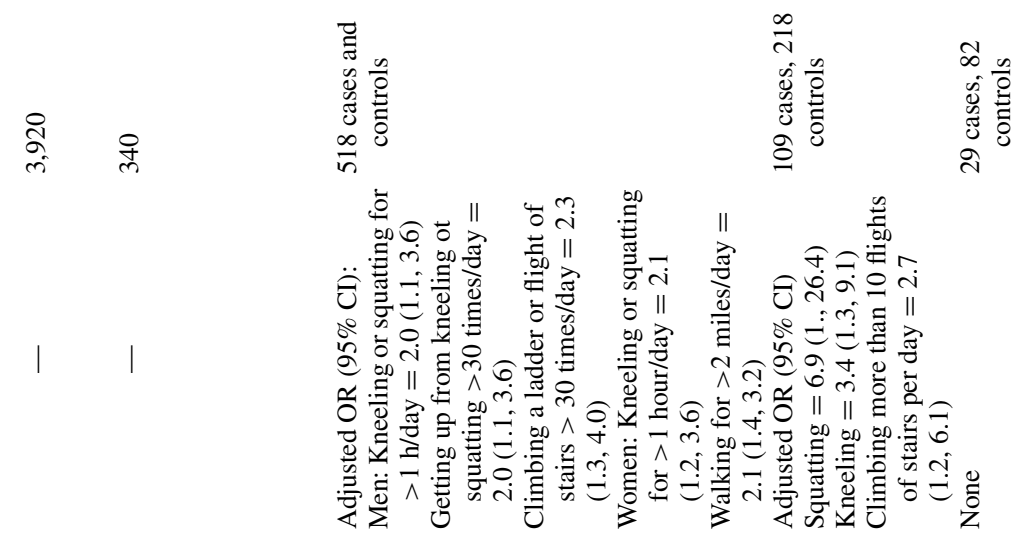

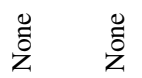

$\stackrel{0}{0}$

$\stackrel{0}{0}$

$\stackrel{\dddot{z}}{\check{z}}$
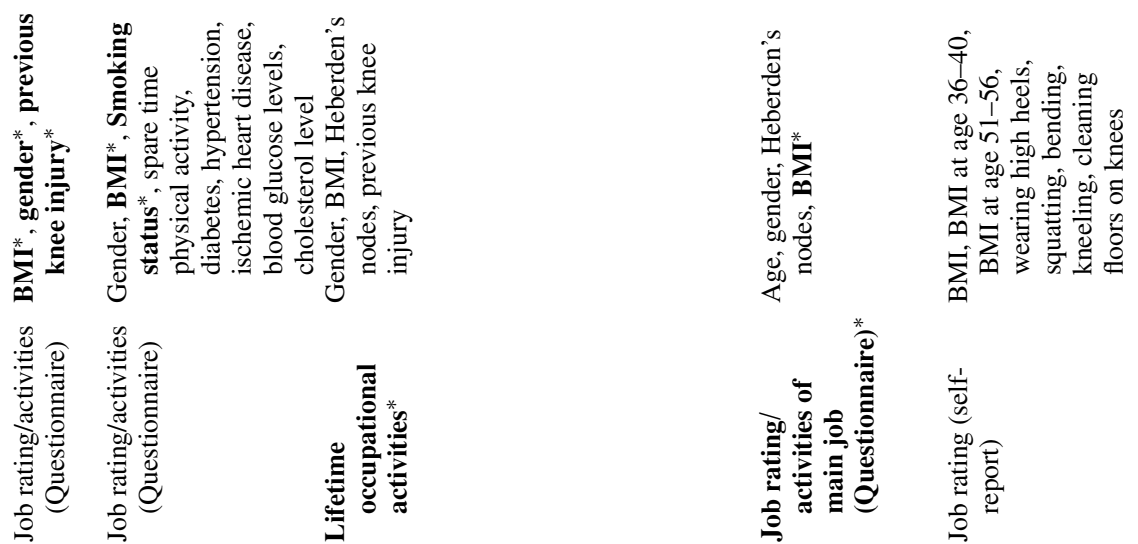

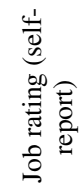
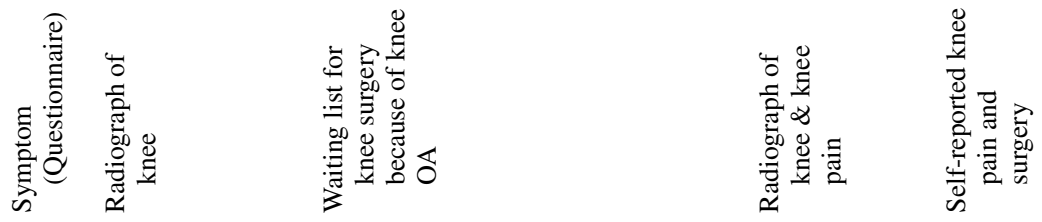

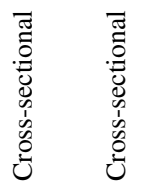

0
0
0
0
0
0
0
0

O
0
0
$\vdots$
0
0
0
0

$\overline{0}$
0
0
0
0
0
0

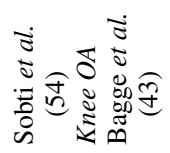

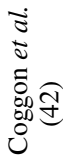

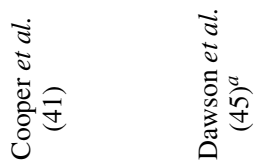




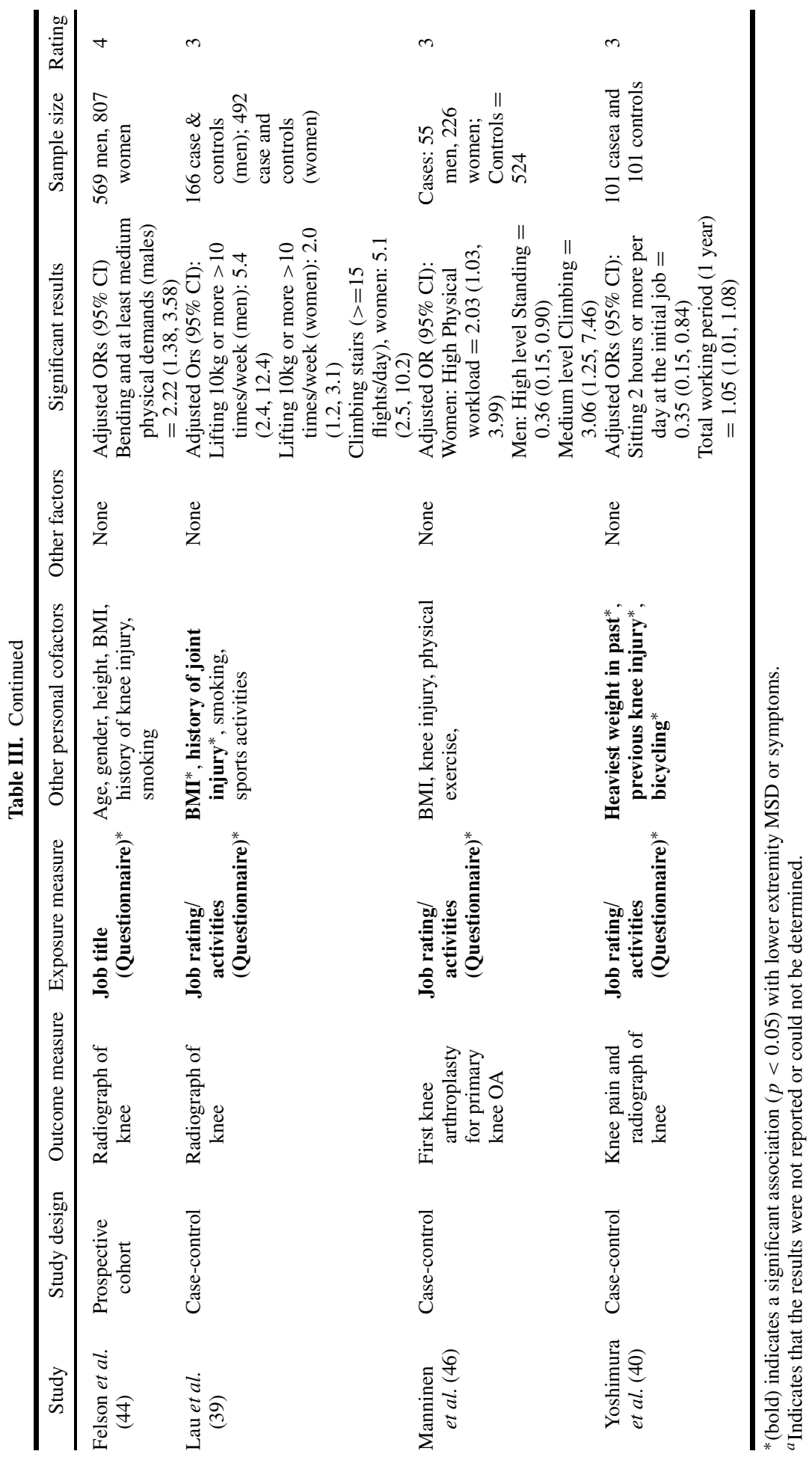


selected for each case from the case's area of residence. Data regarding medical history, current weight, heaviest weight after age 25 , and lifetime history of leisure activities and occupational activities (e.g., sitting, standing, kneeling, squatting, driving, walking, climbing, and lifting greater than $25 \mathrm{~kg}$ ) were obtained through a structured questionnaire. Occupational exposures were obtained for the earliest job held and for the longest job held. In the adjusted analysis, no specific occupational physical activities were positively associated with knee OA, although total working period was associated with knee OA (OR: 1.05 for 1 year; $95 \%$ CI: 1.01, 1.08). Sitting for greater than $2 \mathrm{~h}$ per day in the subjects' initial job was negatively associated with knee OA (OR: 0.35 ; 95\% CI: $0.15,0.84)$. However, as stated by the researchers, relative to a similarly conducted study in Britain (41) the subjects in the Japanese study had a lower BMI, which may have reduced the effects of occupational exposures.

Another population-based case-control study of knee OA among men and women aged 55 and older was performed by Cooper et al. (41). Subjects were registered with a large general practice in Bristol, United Kingdom. Postal surveys were sent to identify those with and without knee pain. All subjects received knee radiography. Cases were defined based upon positive knee radiographs and reported knee pain $(n=109)$; controls had negative knee radiographs and reported no knee pain $(n=218)$. Data on occupational factors were obtained through interview. Height and weight were measured and subjects were examined for the presence of Heberden's nodes. Positive associations were found with obesity and Heberden's nodes (which is a marker of susceptibility to osteoarthritis). Occupational activities such as squatting (OR: 6.9; 95\% CI: 1.8, 26.4), kneeling for greater than 30 min per day (OR: 3.4; 95\% CI: 1.3,9.1), or climbing more than 10 flights of stairs per day (OR: $2.7 ; 95 \%$ CI: 1.2, 6.1), were all positively associated with knee OA (after adjusting for BMI and Heberden's nodes), but heavy lifting was not associated. Examination of the odds ratios suggested an interaction between heavy lifting and kneeling, squatting, or stairclimbing (a measure of repetitive knee flexion), where those who were exposed to kneeling, squatting, or stair-climbing and heavy lifting had 5.4 times the odds of having knee OA than those who were not exposed to either. The researchers also further classified knee OA by the specific location in the knee that was affected. However, the numbers in the analysis became small, and the estimates were less precise. The attempt to distinguish between knee OA by location is helpful in further understanding the relationship between occupational exposures and knee OA. Although self-reported exposures are more descriptive than job title, they may be subject to recall bias, which could overestimate the true association. Also, the researchers looked at the exposures of the subjects' main jobs, which may overlook the contribution of previous job exposures to the development of knee OA.

A case-control study was conducted by Coggon et al. (42) that defined cases as patients who were placed on a waiting list for knee surgery because of knee OA, controls were matched on age and gender and were registered with the same general practitioner as the case. A total of 518 case-control pairs were available for analysis. Both cases and control received a home interview that included questions regarding job history and specific occupational activities. Height and weight measurements were taken and hands were examined by a research nurse for Heberden's nodes. Conditional logistic regression was used in the data analysis. In men, kneeling or squatting for greater than $1 \mathrm{~h}$ per day (OR: $2.0 ; 95 \% \mathrm{CI}$ : 1.1, 3.6), getting up from kneeling or squatting 30 times or more per day (OR: 2.0; 95\% CI: 1.1, 3.5), and climbing a ladder or flight of stairs 30 times or more per day (OR: 2.3; 95\% CI: 1.3, 4.0) were significantly associated with knee OA, after adjustment for BMI, 
Heberden's nodes, and previous knee injury. However in women, walking for greater than 2 miles per day was significantly associated (OR: 2.1; 95\% CI: 1.4, 3.2) but climbing a ladder or flight of stairs and getting up from kneeling or squatting were not. Similar to men, kneeling or squatting was significantly associated with knee OA (OR: 2.1; 95\% CI: 1.2, 3.6). Analysis of the duration of occupational activities was inconclusive for men and women. Odds ratios varied by strata, but there was no clear trend. Interactions were found between occupational lifting and kneeling or squatting and between occupational kneeling or squatting with obesity. As with all case-control studies, this study may suffer from recall bias. Researchers did employ "dummy questions" regarding driving and sitting that may be unrelated to knee OA to assess the degree of bias, which yielded a null association, suggesting minimal recall bias. Also, the researchers restricted their analysis to exposures 10 years before entry into the study, to reduce the effect of workers leaving a job due to early symptoms of knee OA, which gave similar results.

Bagge et al. examined a much older population ( $>70$ years old) that was systematically selected from the older population in Goteberg, as part of a population study 70+ year old People in Goteberg $(n=340)$ (43). Radiographs of the knees were used to ascertain cases and controls. Also, radiographs were taken of the hand joints. Cofactors and occupational activity were obtained through interview. Laboratory data was also available on the subjects. BMI was found to be positively associated with knee OA, while smoking was found to be protective. No associations were found with previously hypothesized variables such as diabetes, hypertension, cholesterol, and blood glucose levels. A significant unadjusted association was found between knee OA and subjects' rating of their occupational physical activities, but disappeared after adjusting for BMI. It is possible that using a population over 70 years may introduce survivor bias.

Felson et al. made use of data from the Framingham Study (44). Knee radiographs were used to assess knee OA (569 men, 807 women). Job titles were used to assess occupational exposure to knee bending and physical demand, based upon ratings in the Dictionary of Occupational Titles. After adjusting for age, BMI, knee injury history, smoking, and education, knee OA was associated with occupational knee bending and at least medium physical demands in males, when compared with men who were not exposed to either (OR: 2.22; 95\% CI: 3.58, 13.8). Few females had jobs that required knee bending and few males with symptomatic knee OA had jobs with knee bending and heavy demands, and thus those relationships could not be examined. Although jobs with only heavy physical demands or knee bending requirement were positively associated with severe radiographic knee OA, these were not significant. A major strength of this study was its longitudinal design, ability to control for important confounders, and its definition of knee OA. However, the exposure information is based upon job titles and the dichotomous knee bending variable provides limited information.

Dawson et al. (45) conducted a case-control study of symptomatic knee OA among women. Subjects were aged 50-70 years and were residents nearby an orthopedic hospital. Cases $(n=29)$ were defined as having reported moderate knee pain on most days in the past month and had been placed on a waiting list for total knee replacement. Controls $(n=82)$ were matched on age and were chosen from the same general practice that the case was registered. Data were collected via interview, using a life-course approach, which uses life events as cues, and triggers to aid subjects' recall of past events/exposures. This method was used to minimize recall bias. Interviewers asked about the wearing of high heels, weight and height at different time periods, smoking, occupation, and occupational 
activities. There was a crude and significant association between symptomatic knee OA and lifting, bending, kneeling, and cleaning floors on knees, but not with squatting or shoes. However, there was no clear dose response relationship and each of the occupational activities was highly correlated with each other, which ultimately led the researchers to decide on including only bending (which was not significant, after adjustment for BMI and wearing high heels). The sample size was very small and limited the power of the study, due to the study's primary aim of testing feasibility of the method. Also, the researchers' main interest was the relationship between wearing high heels and knee OA, thus occupational factors may not have been adequately addressed.

Manninen et al. (46) also conducted a case-control study of knee OA and physical workload. Cases were patients ( $n=55 \mathrm{men} ; n=226$ women) who had undergone their first knee arthroplasty for primary knee OA at a local hospital and were aged 55-75 years old. Referents $(n=524)$ were drawn from the population residing in the same province as the hospital and were age and gender matched. Physical workload was obtained through interview and was assessed for every occupation the subject had until age 49 years. Cumulative exposures to walking, lifting, and driving were calculated and used in the final analysis. Subjects were also asked about prolonged standing, kneeling, or squatting and physical workload (defined by amount of sweating and heart rate). In the multivariate analysis, the association between occupational activities and knee OA was inconsistent by gender. Standing was found to be protective among men (OR: 0.36; 95\% CI: $0.15,0.90)$ and climbing was positively associated with knee OA (OR: 3.06 ; $95 \%$ CI: $1.25,7.46)$. In women, only high physical workload was associated with knee OA (OR: 2.03; 95\% CI: 1.03, 3.99).

Since the latest review (7), Yoshimura et al. (40), Dawson et al. (45), and Manninen et al. (46) have published case-control studies examining the relationship between occupational factors. Yoshimura et al. and Dawson et al. did not show any significant association with occupational factors. The study performed by Yoshimura et al. suggests that the relationship between knee OA and occupational factors may vary according to country. The study by Dawson et al. was inconclusive as the study sample was small and the occupational exposure assessment was poor. Manninen $e t$ al. showed that some occupational factors are significantly associated with knee OA and that standing was protective in men. Specific occupational activities (e.g. kneeling) and heavy weight are thought to increase the stress on the knee which leads to the development of knee OA. Teichtahl et al. (47) have suggested that based upon laboratory studies, biomechanical factors (such as a person's gait) are important determinants in the forces upon the knee. Teichtahl et al. recommend that further studies are required to distinguish whether differences in biomechanics are a result or risk factor for knee OA. The interaction of these individual factors with occupational exposures is important to consider. Also, a laboratory study by Kerrigan et al. (48) suggest that wearing high heels increase forces on the knee joint, when compared to walking barefoot. Therefore attention may need to be given to the types of shoes that people wear, in particular women.

\section{Other Knee Disorders}

Jensen et al. reviewed the literature on occupation and knee disorders (38). Their review focused on knee disorders (osteoarthrosis, bursitis, meniscal lesions, and chondromalacia) and kneeling, squatting, and heavy physical work. The epidemiologic literature regarding meniscal lesions was especially scarce and inadequately controlled for confounding. No 
studies were found on chondromalacia and occupational factors. However, the reviewed literature supported an association between bursitis and kneeling work, while the association between meniscal lesions and occupational factors could not be supported. The conclusions regarding knee OA have been previously mentioned. A large fraction of the studies that were reviewed involved carpet layers and floor layers.

Baker et al. investigated risk factors for meniscal damage through a population based case-control study (49). Cases were patients $(n=243)$ of a local hospital who were diagnosed with meniscal tear. These diagnoses were confirmed via first arthroscopy. Controls were residents $(n=460)$ who were registered at the same general practitioner as the cases. Cases and controls were matched on age and gender. The study population was aged 20-59 years. Data on occupation exposures, and cofactors (e.g. participation in sports, history of knee symptoms, smoking, and alcohol) were obtained through interview. Height, weight, and joint laxity were measured through a physical exam. Higher BMI and occupational kneeling, occupational squatting, lifting, driving, and stair climbing were associated with meniscal lesions after adjustment for social class, joint laxity, and sports participation. Standing and sitting were not associated with meniscal lesions. The researchers conducted separate analyses on degenerative and acute meniscal lesions. Degenertative meniscal lesions were significantly and more strongly associated with occupational kneeling and squatting. This suggests the different mechanisms that occupational activities have on mensical injury. The participation rate among controls was low (28\%). Ascertainment of occupational exposures may be subject to differential recall bias. Also, the cases may not represent all meniscal lesions as they represent only those seen at this particular hospital. Cases may be more likely to seek medical treatment if their jobs are affected by the meniscal lesion. However, the researchers recognized these weaknesses and examined the extent of these shortcomings. In separate studies, the researchers found that the amount of recall bias was likely to be minimal. But that the referral bias may be present in the study and could overestimate the true association. Despite this referral bias, the study design and analysis are well executed. Their results agree with previous literature regarding kneeling and squatting and suggest the importance of looking at meniscal lesions more specifically and investigating the association with driving.

Knee disorders were examined in a community sample of 1404 men, aged 20-59 years (3). The questionnaire asked about lifetime occupational and sporting activities and any history of knee symptoms lasting over $24 \mathrm{~h}$ and meniscectomy. There was a high lifetime prevalence of knee symptoms (54\%), particularly knee pain (45\%). Knee locking had a lifetime prevalence of $9 \%$. Based on a nested-case-control study of 67 men who reported menisectomy and 355 controls, occupational activities such as, kneeling for greater than $1 \mathrm{~h}$ per day, squatting for greater than $1 \mathrm{~h}$ per day, and working in an occupation "likely to involve kneeling or squatting" were significantly associated with meniscal injury (after adjustment for participation in sport). The response rate was rather low (50\%); if subjects are more likely to participate because they experience knee symptoms, the estimated prevalence would be inflated. Also, both outcome and exposure were self-reported. The recall of occupational activities may not be entirely accurate; although the researchers assessed that the possibility of recall bias was low. The researchers provide valuable information regarding the referral bias that occurs when those in physically demanding jobs are more likely to seek medical treatment or services than those that are not.

Chen et al. (50) examined the association between driving and knee pain in a population of taxi drivers $(n=1115)$ in Taipei, Taiwan. Their study was part of an overall larger study 
(Taxi Drivers' Health Study) aimed at assessing cardiovascular disease, job stress, and low back pain. The modified Nordic musculoskeletal questionnaire was used to assess knee pain in the past 12 months. In addition, a self-administered questionnaire was used to collect data on demographics, health behaviors, average number of days they drive per month, average number of hours they drive per day, and average frequency of physical activities (e.g. heavy lifting) in their work and leisure activities. Other factors such as mental health and job dissatisfaction were also measured through questionnaire. Physical examination records were used to collect anthropometric data. Multiple logistic regression was used to analyze the data. After adjustment for age, gender, education, BMI, marital status, income, smoking, professional seniority (in years), days of driving per month, full-time status, frequency of heavy lifting activities, regular exercise, mental health score, and leisure time physical exertion, subjects who drive $8-10 \mathrm{~h}$ per day had an odds ratio of $2.55(95 \%$ CI $1.32,4.94)$ of reporting knee pain when compared to those who drove less than $6 \mathrm{~h}$ per day, while those who drove 10 or more hours per day had an odds ratio of 3.14 (95\% CI 1.62,6.08). Moderate to severe job stress (OR: 1.78; 95\% CI: 1.06, 2.99), low mental health score (OR: 1.77; 95\% CI: 1.26, 2.50), leisure time physical exertion (OR: 1.94; 95\% CI: 1.12, 3.34), and registration type (individual practice vs. affiliated with taxicab company or cooperative practice, the referent group) (OR: 1.60; 95\% CI: 1.09, 2.35) were also significantly associated with selfreported knee pain. Bending/twisting and high job dissatisfaction were not significantly associated with knee pain. Although the study is limited by its cross-sectional design and self-reported job exposure and outcome, the researchers' analysis of the data was extremely detailed and thorough, suggesting that their association between driving and knee pain is not an artifact. Furthermore, the researchers assessed the validity of the self-reported driving hours, by comparing self-reported numbers with those retrieved from structured interviews and diary records. The self-reported measures tended to overestimate the "actual" by $0.9 \mathrm{~h}$ but were independent of knee pain.

Miranda et al. (51) conducted a prospective study on forestry workers with and without knee pain to examine risk factors for incident and persistent knee pain. Both exposure and outcome were assessed through questionnaire. A 1-year follow-up questionnaire was completed by subjects. Subjects were asked about the frequency of specific work activities, psychosocial factors at work, physical exercise, smoking, previous knee injury, mental stress, age, and BMI. Previous knee injury was found to be significantly associated with incident knee pain (OR: 2.7; 95\% CI: $1.8,4.1$ ) as was BMI. However, no work-related factors were significantly associated with incident knee pain. Job dissatisfaction and twisting movement of the trunk were associated with persistent severe knee pain, however these results were not reported. The prospective nature of the study is clearly a strength and many of the questions regarding workload had been previously validated by observed estimations.

A previously mentioned cross-sectional study of apprentice construction workers $(n=$ 996) by Merlino et al. attempted to estimate the prevalence of musculoskeletal symptoms (18). Based upon a modification of the Standardized Nordic Questionnaire, 38.4\% of the subjects reported knee symptoms within the past 12 months. Also working in same position (OR: $2.11 ; 95 \%$ CI: 1.52, 2.93) and inclement working condition (OR: 1.47; 95\% CI: $1.04,2.07)$ were associated with knee symptoms. A significant trend existed between years worked and prevalence of knee symptoms. In addition to the limitation aforementioned, the workers were not asked to rate the frequency of the work activities but how much they thought each work activity contributed to MS symptoms, which may have introduced a high amount of recall bias. 
Jensen et al. investigated the prevalence of knee disorders and complaints in floor layers and carpenters $(n=1488)$ (52). Observations of knee straining work while performing certain tasks were used primarily to validate the exposure differential by job categories. Knee complaints, occupational history, history of knee injuries, age, height, and weight were obtained through questionnaire. Medical examinations were used to determine the reproducibility of physical signs of certain knee disorders (e.g. Lachmann's test, bursitis). However, the reproducibility was low ( $\kappa$ ranging from 0.05 for bursitis to 030 for retropatellar crepitation). Moderate reproducibility was demonstrated for knee hyperkeratoses $(\kappa=0.55)$, which the researchers concluded has little clinical relevance. Results of the videotape analysis confirmed significant differences between job categories in the amount of knee straining work-floor layers (high exposure), carpenters (medium exposure), and compositors (low/no exposure). Floor layers had the highest prevalence of knee complaints, followed by carpenters, and the compositors had the lowest prevalence of knee complaints. Floor layers and carpenters that are not presently working in their trade reported more knee complaints than compositors, but less than those who are presently working in their respective trades. They did not find any significant effects of age, seniority, BMI, and knee-straining sports. Although the cross-sectional design of this study is a limitation, the study's exposure assessment is a major strength because of its ability to describe the amount of knee straining work in a job category rather than relying on job title alone.

Lagerstrom et al. also investigated MS symptoms in nursing personnel $(n=688)$ (53). However, the cross-sectional study focused on the neck, shoulders, low back, hands, and knees. The questionnaire asked about psychosocial factors, age, and BMI. The job exposure at work was classified according to the type of ward in which the nurses worked (some departments require more patient handling than others). It was found that knee symptoms were related to increasing age and high BMI. No significant associations were found between type of ward (the job "exposure"), psychosocial factors, and knee symptoms.

deZwart et al. found significant increases of approximately 3\% in the prevalence of knee complaints in the heavy physical demand group ("exposed") and in all age strata (except for the oldest, 50-59 years) over a 4-year period, based upon repeated surveys of male employees in The Netherlands ( $n=7324$ exposed; 4686 unexposed) (16).

Lemasters et al. studied construction workers $(n=490)$ through a questionnaire $(23)$. Physical examinations were used to validate the self-reported knee disorders, which showed that subjects correctly recalled their knee disorders. Knee disorders, which included symptoms, were shown to be associated with duration as a carpenter (OR: 3.5; 95\% CI: 1.3 , 9.2), feeling exhausted at the end of day (OR: 1.8; 95\% CI: 1.1, 3.1), and minimal schedule influence (OR: $2.3 ; 95 \%$ CI: 1.2, 4.1). Although carpentry work is well known for its heavy physical demands, the relationship with specific work tasks was not explored in this study. The study does show the importance of psychosocial factors at work and their relation to MS symptoms and disorders. Also, the researchers conducted a validity and reliability analysis of their survey, which showed that subjects correctly recalled knee disorders (validated by a physical examination) and reliably recalled both their symptoms and knee disorders.

Sobti et al. conducted a survey of a large population of retired postal workers $(n=$ 3920) (54). The study aimed to address the relationship between physical activity in the workplace and subsequent musculoskeletal pain. Subjects reported the frequency of certain tasks (e.g. lifting, climbing stairs). A mild association was seen between BMI and knee symptoms (OR: $1.54 ; 95 \%$ CI: $1.27,1.87)$ with a significant trend $(p<0.001)$. Women 
also reported more knee symptoms than men. However, no significant association between workplace factors and knee MS pain or knee replacement were found. There may be recall bias in the reporting of job exposures. Additionally, the accuracy of the exposures may be reduced, as retired workers may not correctly recall their exposures.

O'Reilly et al. also conducted a cross-sectional study of a geographically defined population (4). Postal surveys were used to assess the prevalence of knee pain among men and women aged 40-80 in relation to self-reported occupation, which resulted in a response rate of $82 \%(n=3326)$. The occupation among males associated with the highest prevalence was carpentry $(60.9 \%, n=15)$, while for females it was sales assistant $(37.1 \%$, $n=54)$. For women, occupations with the lowest prevalence of knee pain were teachers $(18.9 \%, n=107)$, cleaner $(21.9 \%, n=117)$, and secretary $(23.4 \%, n=94)$. Managers $(22.0 \%, n=186)$, police/security officers $(23.8 \%, n=43)$, and teachers $(20.8 \%, n=72)$ had the lowest prevalence of knee pain in men. Thus, in men, carpentry had approximately a three times greater prevalence of knee pain than teachers. In women, the relative difference was not as large, with sales assistance having slightly twice the prevalence of knee pain relative to teachers. Significant adjusted (age, gender, BMI, social class, smoking history, and mental health score) odds ratios were found between knee pain and carpentry, mining, and construction occupations (combined OR: 1.8; 95\% CI: 1.4, 2.4).

The knee OA literature shows a mostly consistent relationship between knee bending and knee OA. Other knee disorders (e.g. meniscal lesions, meniscal tear) were associated with occupational kneeling and squatting and possibly standing (3). Knee symptoms were associated with awkward posture, "knee straining work," work history (duration as a carpenter), and "heavy physical demands." More detailed exposure data is needed to identify the specific occupational exposures that are related to knee symptoms. Also, the recent study by Chen et al. (50) strongly suggests that driving be further studied in order to better understand its relationship with knee pain.

\section{HIP/THIGHS}

Similar to the literature on knee disorders, most of the literature on the hip and thigh area is largely focused on osteoarthritis of the hip. Three case-control studies of hip OA and seven studies of hip pain and symptoms are further discussed. Some of the results of the hip pain/symptom studies may be helpful in predicting hip OA as hip pain/symptoms may be an intermediate factor in the causal pathway between occupational exposure and hip OA. (Table IV).

\section{Osteoarthritis}

Three reviews of the literature on osteoarthritis of the hip have been done (5-7). Maetzel et al. concluded that epidemiologic studies show an association between "workrelated exposure" (i.e. standing, walking, lifting, and job title) and hip OA in men, and only a weak relationship in farmers (5). None of the studies reviewed attempted to investigate occupational factors and hip OA in females. A major weakness of the studies was the measurement of occupational exposures, which were primarily based upon job title and subject to recall bias (i.e. occupational exposure obtained through interview). Schouten 


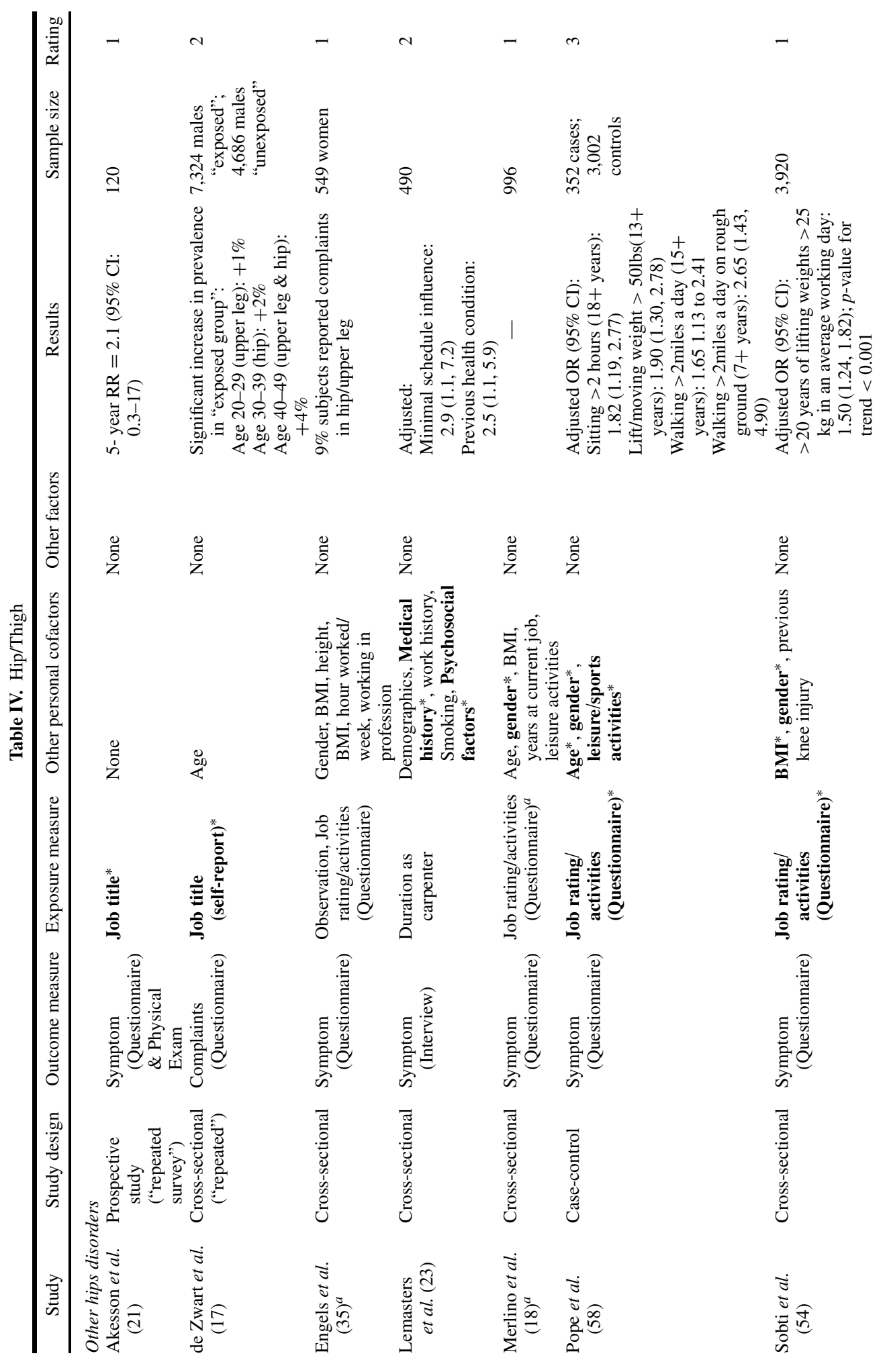




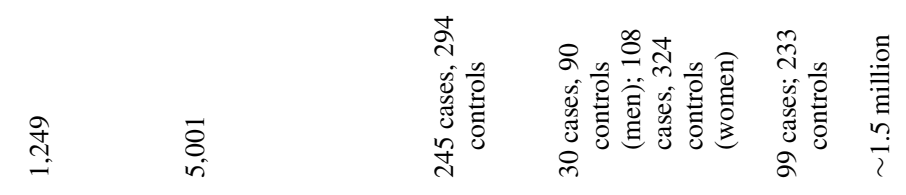

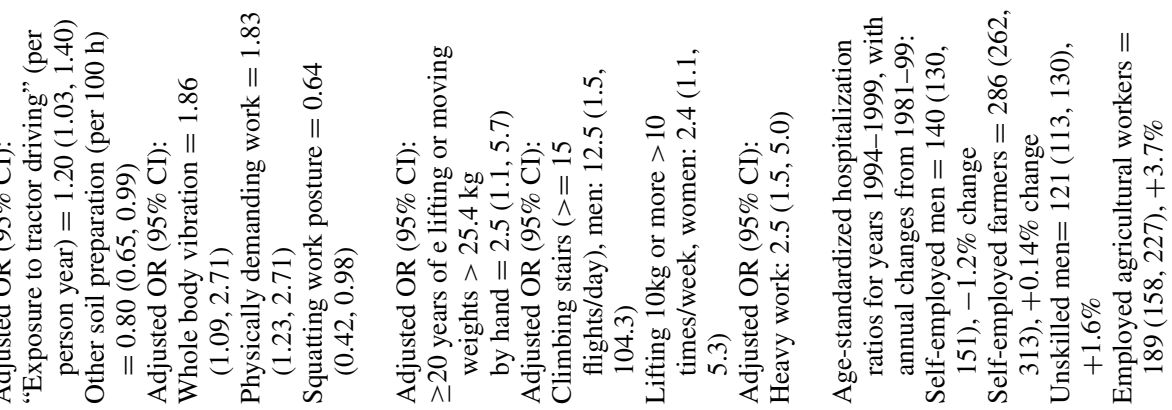

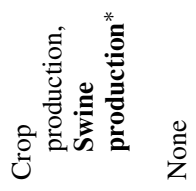
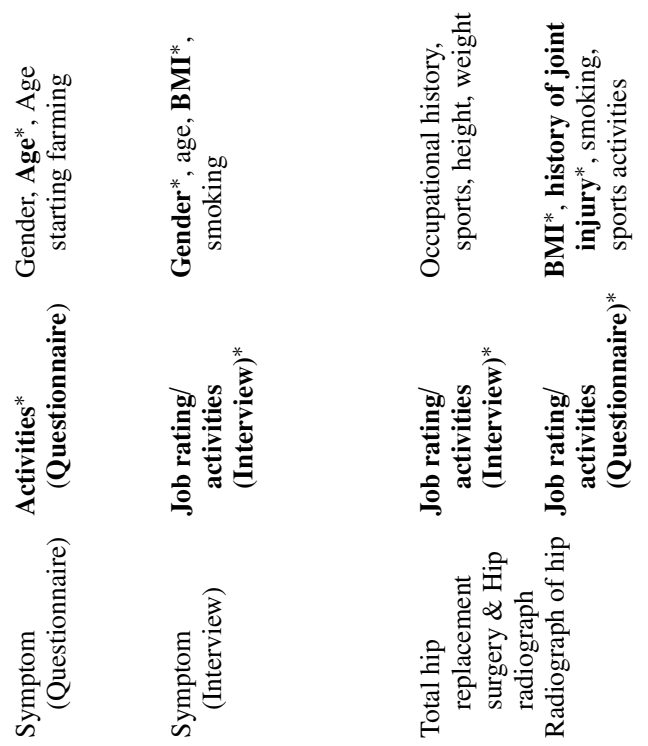

Z
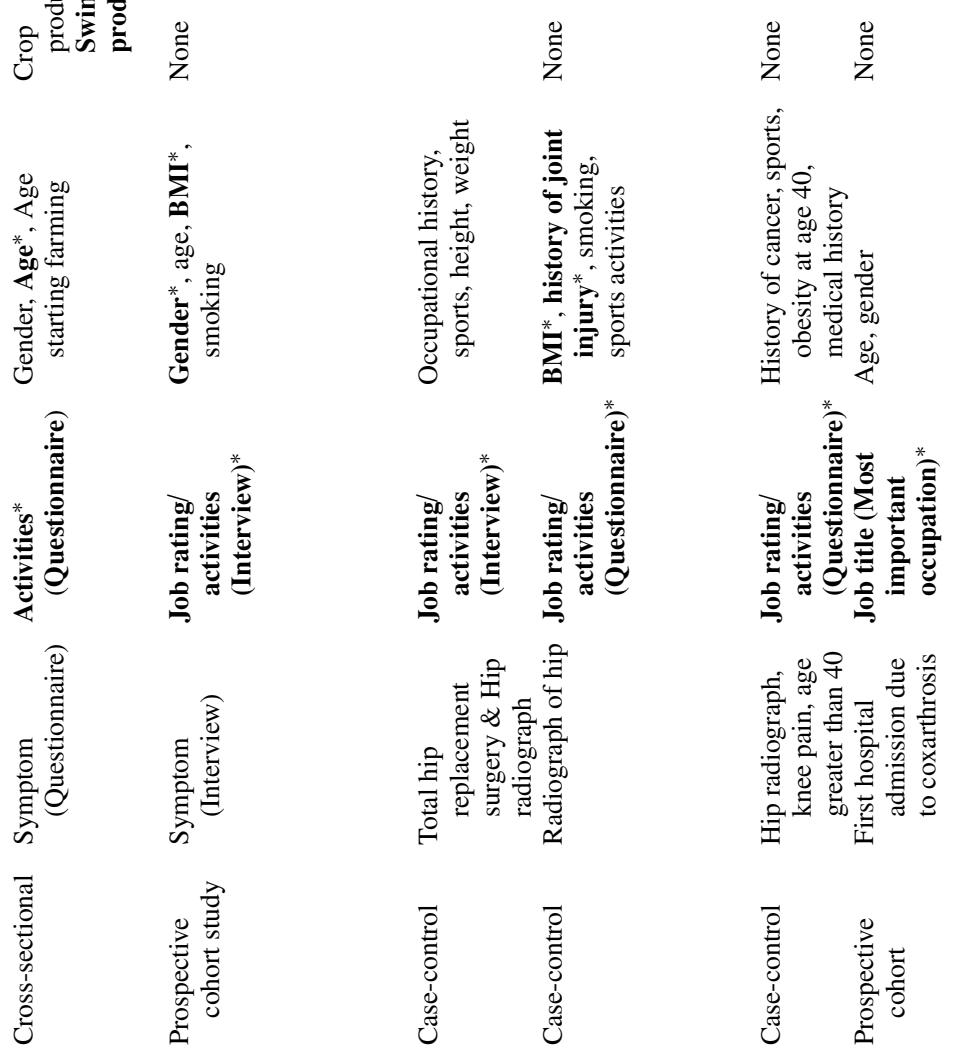

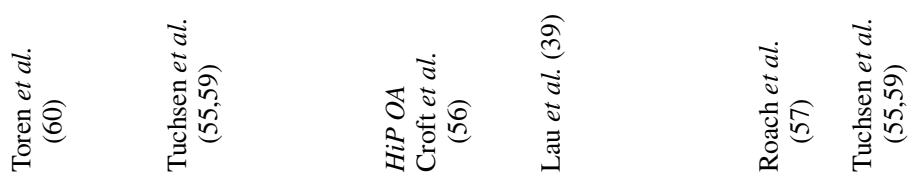

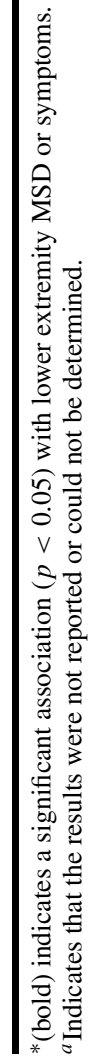


et al. (7) attempted to update the earlier review, by reviewing articles published in 2000 and 2001, and chose studies that had 1) a study design with a comparison group 2) a mechanical influence of occupation or occupational factors 3) no sporting activities could be studied as main determinants 4) OA was defined according to the American College of Rheumatology criteria or a radiographic scoring system. The two identified studies showed a significant relationship between heavy lifting and hip OA in both men and women, and climbing stairs was associated with hip OA in men. Both studies had greater than 103 cases, but both studies focused on severe hip OA (hospital cases and being listed from hip arthroplasty), which may have introduced selection bias. But Schouten et al. concluded that the results provide additional evidence for the relationship between work factors and hip OA. Finally, Lievense et al. also evaluated the hip OA literature and concluded that there is "moderate" evidence for the effect of heavy physical workload and occurrence of hip OA (6).

Tuchsen et al. (55) performed a prospective cohort study of four cohorts of employed Danish men, using the population census and national patient register (which includes data on all admissions to public and private Danish hospitals). Occupation and first hospital admission for coxarthrosis were obtained using these data sources. Age-standardized hospitalization ratios (SHR) were calculated using age-specific incidence rates for employed men in Denmark. The study population was large $(\sim 1.5$ million $)$ and the follow-up period was approximately 5 years. The researchers found significant SHRs for agricultural occupations in all four cohorts and occupations such as machine operators (SHR: 207; 118.3, 336.2), wood product machine operators (SHR: 205; 119.3, 327.9), and waiters and bartenders (SHR: 204; 102.0, 365.6). The study had the advantages of a large study population and prospective design. The outcome assessment was nearly complete. However, the "exposure" was just job title. Thus future studies may want to address the specific work activities that put workers in a particular occupational category at higher risk of hip OA.

An age- and gender-matched case-control study of hospital patients (138 cases, 414 controls) with osteoarthritis of the hip and knee (39) found that frequently climbing stairs at work was significantly associated with hip OA in only men (OR: 12.5; 95\% CI: 1.5, 104.3) while lifting $10 \mathrm{~kg}$ or more for more than 10 times per week was significantly associated with hip OA in only women (OR: 2.4; $95 \%$ CI: 1.1, 5.3). History of joint injury was significant in both genders, and interestingly body height was associated with hip OA in women.

Another case-control study was done on osteoarthritis of the hip and occupational activity in males (56). The cases and controls were drawn from a population who had outpatient intravenous urograms. Cases $(n=245)$ were those who had a total hip replacement or whose radiography displayed a minimum joint space equal to or less than $2.5 \mathrm{~mm}$. Controls $(n=294)$ were those who had a greater minimal joint space and had not undergone total hip replacement. Occupation and occupational exposures were assessed through interview. After adjusting for age and hospital group, a nonsignificant association was found between severe cases of hip OA and more than 10 years of employment in farm and/or agricultural work (OR: 2.0; 95\% CI: 0.9, 4.4). The strongest association found was between severe cases and greater than 20 years of exposure to lifting or moving weights greater than $25.4 \mathrm{~kg}$ by hand (OR: $2.5 ; 95 \% \mathrm{CI}: 1.1,5.7)$ and with greater than 20 years of exposure to walking more than 2 miles per day (OR: 1.9; 95\% CI: 0.9, 4.1). No significant associations were found between hip OA and BMI, which suggests that this study population may not accurately represent the working population. Despite the nonsignificant associations, the study used patients with intravenous urograms, which reduced the bias that occurs when patients present themselves with hip symptoms or for hip treatment. 
Roach et al. conducted a similar case-control study of hip OA (57). Cases were selected based on hip pain, hip radiographs, and greater than 40 years of age $(n=99)$. Controls were taken from the patient population who had intravenous pyelograms because the hip joint was visible $(n=233)$. Occupational exposure was determined through self-reported number of years in a job that was categorized into occupational categories. Based on the number of years reported for each occupational category, the subjects were classified into the final occupational exposure categories of light and heavy work. Although a significant statistical association was found between history of cancer and hip OA, history of cancer was not significantly related to work exposure. Adjusted associations demonstrated an increased odds of hip OA among men in "heavy work" (OR: 2.5; 95\% CI: 1.5, 5.0). A significant trend was found between increasing levels of exposure and hip OA. The subjects were drawn from the patient population of the Veterans Affair system and may not be comparable to the general population. However, as stated by the researchers, use of the VA likely eliminated selection biases that occur when hip OA cases do not seek treatment due to economic reasons.

\section{Other Hip Disorders}

Hip pain was examined by Pope et al. in relation to occupational activities (58). A population based case-control study was conducted. Cases and controls were identified through general practices in Cheshire, United Kingdom. Cases were defined as subjects who responded positively in a questionnaire asking about hip pain in the past month that lasted more than $24 \mathrm{~h}(n=352)$. All other subjects were controls $(n=3002)$. Occupational exposures for each job held were also obtained through the questionnaire and were dichotomous variables (e.g. standing for at least $2 \mathrm{~h}$ without a break, sitting for at least $2 \mathrm{~h}$ without a break). A cumulative exposure was calculated by summing up the number of years a subject was in a job that involved the specified work activity. Lifetime participation in specific sporting activities (e.g., swimming, track/field, cycling, etc.) was also obtained. After adjustment for age and gender, the researchers found a significant association between reported hip pain and the following occupational physical demands: sitting for greater than $2 \mathrm{~h}$ without a break (OR: 1.94; 95\% CI: 1.28, 2.95), lifting/moving weight greater than $50 \mathrm{lbs}$ for greater than 13 years (OR: 1.90; 95\% CI: 1.30, 2.78), walking greater than 2 miles per day for greater than 15 years (OR: 1.65; 95\% CI: $1.13,2.41$ ), and walking greater than 2 miles per day on rough ground for greater than 7 years (OR: 2.65; 95\% CI: 1.43, 4.90). The researchers did not adjust for injury history. BMI was not included in the adjustment as it is subject to reporting error by subjects, and in this study, it may have not been appropriate to adjust for a factor that may be a consequence of the outcome. Also, the researchers defined cases as those who experienced hip pain during the past month lasting at least $24 \mathrm{~h}$, and controls as those who did not. This may have resulted in a misclassification of cases as controls, resulting in an underestimation of the true associations.

Akesson et al. surveyed female dental personnel twice, at the beginning and the end of a 5-year interval (21). The dental personnel were selected from the local public dental care service. They were compared with a sample of 30 female medical nurses who were employed at hospitals in the same area. The Nordic questionnaire was used to assess symptoms. Also, pain and functional disturbances were also measured through questionnaires. These instruments were used again at the end of the 5 years to assess changes. A physical 
examination was also used to diagnose disorders specific to the hips (as well as other areas, but excluding any other lower extremity diagnoses) and assess symptoms and/or positive signs ("findings"). The diagnosis was made based upon preset criteria (sacroiliac pain, piriformis syndrome, trochanteritis, and Coxarthrosis). At baseline, there was a higher prevalence of hip pain, musculoskeletal symptoms of the hip, diagnoses and clinical finding of the hip in dental personnel, but only the prevalence of clinical findings in the hip was significantly higher in referents. The 5-year unadjusted relative risk of developing MS symptoms in the hip was positive $(\mathrm{RR}=2.1)$ but nonsignificant. However, there was a wide variation in the diagnoses. Furthermore, the subject population is rather small (90 dental personnel, 30 referents) and may have limited power to detect true differences. Also, the focus on the female gender limits the generalizability of the study. Beyond anecdotal observations, there were no data on job exposure or psychosocial variables. There was lack of adjustment for BMI and other possible confounders. Additionally, comparing dental personnel with nurses may not be a valid comparison. It was not made clear in what respects the nurses' work was "physically light" compared to dental workers. A major strength of the study was its prospective design, allowing the observation of incident MS symptoms, pain, findings, and diagnoses. The researchers' use of physician diagnoses and clinical findings provide a richer and helpful description of the disease experience of dental personnel. In addition, the combination of questionnaires and physical examination allows the evaluation of various screening tools and gives a more complete assessment of disease in the study population.

In the Merlino et al. (18) study of apprentice construction workers $(n=996)$, women reported significantly more musculoskeletal symptoms $(23.9 \%, n=16)$ in the hip/thigh than men $(8.4 \%, n=78)$. But this association with gender was not adjusted for work history or the specific occupation within the construction industry.

The deZwart et al. study on changes in musculoskeletal complaints found a greater increase in hip and upper leg complaints in the heavy physical work demand group compared to the mental work/control group (16). The significantly greater change in hip complaints was limited to the 30-39-year age stratum ( $+2 \%$ vs. $+1 \%$ change), and 40-49-year age stratum ( $+4 \%$ vs. no change). However for upper leg complaints, the significantly greater changes were limited to the 20-29-year age stratum (+1\% vs. no change) and 40-49-year age stratum ( $+4 \%$ vs. no change). The poor assessment of exposure and lack of data on additional cofactors and confounders limit the utility of the results.

Sobti et al. found significant associations between higher BMI, increasing years of lifting weights (greater than $25 \mathrm{~kg}$ ), and the hip symptoms in a survey of retired postal workers $(n=3920)$ (54). Those who spent more than 20 years of performing heavy lifting experienced a 1.5 higher odds (95\% CI: 1.24, 1.82) of reporting hip pain or stiffness than those who were not exposed. There was a significant trend as well $(p<0.001)$. Additionally, women reported more hip symptoms than men (30.4\% in women, $19.9 \%$ in men).

Tuchsen et al. conducted a prospective study on a population of employed persons $(n=5001)$ (59). Surveys were conducted at the beginning and end of a 5-year period. Interviewers asked subjects regarding hip pain in the past 12 months as well as occupational exposure to activities such as whole body vibration, squatting, and heavy lifting. A total of 223 incident cases of hip pain were observed over the 5-year period. Women developed hip pain twice as often as men. BMI was also associated with pain. Whole-body vibration (OR: 1.86; 95\% CI: 1.09, 3.18), squatting (OR: 0.64; 95\% CI: $0.42,0.98$ ), and physically demanding work were all found to be associated with hip pain. 
Toren et al. (60) conducted a cross-sectional survey of farmers in a county of Sweden in order to examine the association between tractor-driving hours and self-reported hip symptoms. Questionnaires were mailed to all farms with acreage of more than 10 ha. Sixty percent of the farms responded and 1249 farmers were included in the analysis. The questionnaire asked about demographic factors and exposure factors (farm and tractordriving data). Hip symptoms were obtained using the Standardized Nordic Questionnaire. The average annual tractor-driving time in the previous 10 years and the number of years the farmers had regularly driven tractors in agricultural work were combined into a single variable by multiplying the number of years and the number of hours. In a multivariable logistic regression model, there was an OR of 1.20 (95\% CI: 1.03, 1.40) for each "person year" of tractor-driving. "Other soil preparation" (per $100 \mathrm{~h}$ ) was protective (OR: 0.80; 95\% CI: $0.65,0.99)$. The response rate was low and weight and height were not included in the analysis. The study attempted to quantify the tractor-driving exposure among farmers as well as investigate other associations between farm work and hip symptoms.

Lemasters et al. found that hip disorders were associated with minimal schedule influence (OR: 2.5 ; 95\% CI: 2.2, 5.9) in construction workers, which suggests an effect of psychosocial factors (23).

Engels et al. found a prevalence of MS complaints in the hip/upper leg of $9 \%$ of nursing staff in nursing homes $(n=628)(35)$.

The case-control study design has been used by researchers (55-57) to examine hip OA. However, a possible weakness of some of these studies is the possibility of selection bias. A radiograph is necessary in order to validly diagnose hip OA. Hence many studies have been conducted among hospital patients who have sought medical care for their hip OA and already have OA. However, the medical seeking behavior could be driven by the patient's occupational physical demands or other unknown factors. Another method of inexpensively obtaining hip radiographs is to assess patients who have a radiograph of the hip performed as part of the intravenous urogram or pyelograms. This method eliminates possible bias of the medical-care seeking behaviors in hip OA patients.

Most of the literature on the hip and occupational factors has focused on hip OA. The studies suggest a role of occupational mechanical factors in the development of hip pain/symptoms and hip OA. But a more detailed occupational exposure assessment is required to clearly establish which occupational activities are associated with hip MSDs and symptoms.

\section{LOWER EXTREMITY (GENERAL)}

Symptoms and complaints of the lower extremities were studied generally in four epidemiologic studies. All of the studies were cross-sectional, but are important due to the scarcity of literature regarding any lower extremity symptoms and complaints. Additionally, they illustrate the high prevalence of lower extremity complaints and symptoms in occupational populations. (Table V).

Engels et al. determined the prevalence of musculoskeletal complaints in the nursing profession through questionnaires distributed in four nursing homes $(n=846)(22)$. The questionnaires also addressed demographic information, physical stress, and psychosocial factors. Although questions were asked about the specific sites of the lower extremity, the analysis combined all lower extremity complaints. The prevalence of lower extremity complaints was $16 \%(n=133)$ and with the majority of the complaints focused on the 


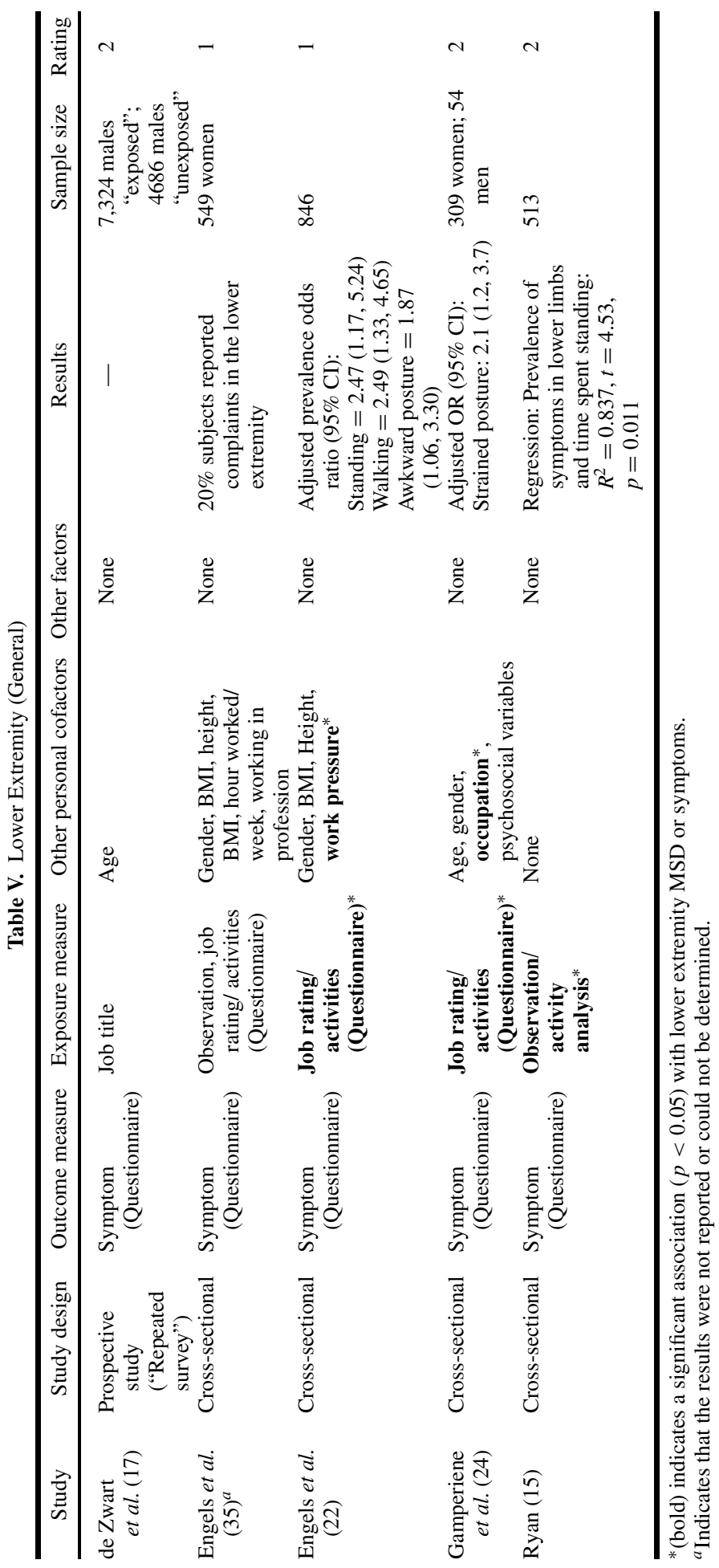


knees. Forty-seven percent of the subjects $(n=63)$ who had lower extremity complaints sought medical care for the symptoms. Strong associations were also found with psychosocial factors and increased physical stress (e.g. prolonged standing, walking, and awkward posture). Like many of the epidemiologic studies, exposure was based on self-report.

Engels et al. performed another study on the prevalence of MS complaints of nurses in nursing homes ( $n=549$ ) (35). Questionnaires were used to assess age, BMI, and work history. This study included an assessment of the physical workload of a sample of 36 subjects. The Ovako Working Posture Analyzing System (OWAS) was used to categorize the position of different body parts of 10 predetermined tasks. There were seven categories of posture for the legs. It was found that nurses spend $65 \%$ of their standing with both legs straight or one leg straight. A major strength of this study was the attempt to categorize exposures according to observation. However, the study had no referent group and the analysis was restricted to women. Due to the aggregate nature of the exposure data, no determination can be made about whether the exposure in fact caused the complaints.

Gamperiene et al. described the prevalence of self-reported MS complaints among workers in the spinning industry $(n=363)(24)$. Occupational exposure was determined through self-report; workers rated specific ergonomic risk factors. Although the researchers reported high prevalences of MS complaints of the knee/lower leg (39.4\%) and ankle/foot (30.9\%) occurring in the past year, the analysis combined the MS complaints into "leg." The study found that women reported more leg complaints than men (65\% vs. 38.9\%). After adjustment for age and occupational group, there was a significant association between "strained posture" and leg complaints. But the job factors were treated as dichotomous variables (yes/no) and were self-reported.

DeZwart et al. surveyed a sample of male employees in The Netherlands at the beginning and end of a 4-year period. After the 4-year period, the researchers found that, compared with the "low physical demand group," there were significantly more subjects who reported lower limb symptoms at both time points, and significantly more "new cases" of lower limb complaints (ie. Subjects who were symptom free at the beginning of the 4-year period but later developed musculoskeletal complaints). This was observed in all age groups.

Epidemiologic studies that have studied general lower extremity symptoms have shown a significant relationship with occupational factors. These studies have created broad exposure and outcome categories. It is difficult to gain further understanding into specific issues, and thus the studies can only provide an overall description of the problem.

\section{DISCUSSION}

\section{Study Design}

The epidemiologic literature on work-related lower extremity disorders consists mostly of cross-sectional studies. The strengths of the cross-sectional design lie in the ease and speed with which data can be collected on large populations and possible salient risk factors can be identified. However, a large weakness of the cross-sectional design is in its inability to further investigate causal relationships between outcome and exposure. For example, the association between BMI and lower extremity musculoskeletal complaints has been consistent, but little is known about the precise roles of BMI and occupational exposure 
and lower extremity musculoskeletal outcomes, namely the temporal relationships between BMI and occupational exposure and lower extremity complaints.

Some study designs have used repeated surveys to describe the relationship between workplace factors and lower extremity musculoskeletal disorders and symptoms. However, these study designs have limited value as they often do not quantify individual exposure data, and are not able to calculate person-time.

The stronger study designs (case-control and cohort studies) have been primarily of osteoarthritis of the hip and knee. Furthermore, most of the studies have focused on surgical (i.e. severe) cases of OA, which may introduce bias (if those who have severe disease are different from those who do not) or may exclude those who change jobs before the OA progresses to the severe stage.

\section{Population}

The epidemiologic literature on work-related lower extremity musculoskeletal disorders has examined particular occupational groups. Those studies have largely been in response to observably high exposures (e.g., carpet layers) and high prevalence of overall musculoskeletal symptoms (e.g. nursing). Certain occupational groups have lists of their workers (e.g., unionized workers), which make it easy to create a complete sampling frame. Also, some occupational groups are often concentrated in certain areas (e.g. hospitals), which also facilitate carrying out the study.

However, certain occupational groups have unique characteristics that prevent results from being generalizable. For example, floor layers spend a large portion of their job on their knees $(38,52)$. An association was found between their occupational exposure and knee complaints. But very few workers outside of floor layers will experience such a high exposure to the knees. Thus, although this association is important for floor layers, it may not be possible to generalize this association to other occupations. This may be true for studies that restricted their study population to a particular gender. Also, the correlation between gender and certain occupations may make it difficult to tease out gender and occupational effects.

As mentioned, the selection of cases and controls in hip OA studies may limit the generalizability or bias the results because of the significant differences in the patient and occupational populations.

\section{Exposure}

A primary reason for the limited generalizability and validity of the epidemiologic literature is the manner of exposure measurement. The occupational exposures were primarily obtained through self-report and job titles. Some self-reported occupational exposures (eg. whole body postures) are reproducible and valid $(61,62)$, but not all, in particular lifting and awkward postures. Thus for certain MSDs (e.g., Standing and VV) self-reported exposures may be quite useful, but for others (e.g., knee OA and lifting) a better metric is needed. Miranda et al. validated their questionnaire with observation. However, for some MSDs and symptoms, the cumulative/lifetime exposure is important and thus the accuracy of self-reporting of these exposures remains undetermined. Dawson et al. attempted to use a life-course approach to facilitate subjects' recall of past occupational exposures (45). 
The use of job titles as exposure classification is also subject to error. It is common that job titles do not represent the true exposure of the worker. Workers with the same job title can have different exposures based upon the workplace's particular needs. Thus use of the job title can misclassify a worker's exposure and result in biasing the true association toward the null. Despite these shortcomings of occupational title, some studies have found associations with occupation, strongly suggesting that further investigation is needed as to what aspects of the occupation are hazardous. Jensen et al. used videotape to validate the amount of knee straining work in each job title (52). Ryan also made use of observation to characterize the amount of time spent performing certain tasks (15). But neither Jensen et al. (52) nor Ryan (15) actually measured the severity and specific characteristics of the exposures (e.g., pressure on the feet), which make it difficult to design interventions.

Engels et al. used heart rate as a measure of physical workload, in addition to perceived exertion (35). But heart rate has limited validity for examining biomechanical exposures specifically related to lower extremity musculoskeletal disorders. The study also used observations to characterize postures in the nursing staff using the OWAS Analyzing system.

Keyserling et al. developed a "one page checklist" for determining the presence of ergonomics risk factors associated with awkward posture of the lower extremities (as well as for the neck and trunk) (63). The results of the checklist were found to be comparable to the results generated by experienced ergonomists. The checklist represents a more objective method of characterizing exposures, but the utility in epidemiologic studies is unknown.

\section{Outcome Measurement}

The outcomes ranged from osteoarthritis, plantar fascitis, bursitis, meniscal lesions, and venous disease to fatigue, symptoms, and complaints. Studies investigating hip and knee disorders were more common than those of the lower legs, ankles, and feet. The relationship between osteoarthritis of the hip and occupational risk factors has been studied through mostly cross-sectional studies; the literature has also been previously reviewed (5-7).

Osteoarthritis of the hip and knee were often ascertained by radiographs read by investigators. However, the outcome of osteoarthritis was also determined by having had (or being on a list for) arthroplasty, surgical treatment of osteoarthritis, or physician diagnosis. These were not always validated through radiographs by the researchers.

Since Jensen and Eenberg's review, few studies have investigated bursitis and occupational factors (38). Jensen et al. ascertained bursitis through physical examination, but the reproducibility was low $(\kappa=.05)(52)$. Previous studies by Kivimaki (64) and Myllymaki et al. (65) used ultrasonographic examinations to determine fluid accumulation in the bursa. However, the correlation between knee pain and acute bursitis (as defined by ultrasound) was poor. Also, there was poor correlation with previously reported bursitis and ultrasonographic changes in the bursa.

The ascertainment of pain, symptoms, and fatigue were mostly obtained through selfreport, including the use of the Nordic Questionnaire and Borg-10 scale. The questions pertained to symptom frequency, severity, onset and duration, and included questions regarding fatigue. 


\section{Confounding}

Overall, many epidemiologic studies have adequately controlled for certain personal cofactors such as age, gender, and BMI in their analyses. However, factors such as work history and history of joint injury were not always adequately controlled.

However, there is evidence that it is not solely occupational physical activities that can lead to musculoskeletal disorders and symptoms. Laboratory studies have shown that the type of flooring and shoes worn may affect the development of fatigue and discomfort (25). Also, genetics, age, BMI, gender, and smoking complicate the relationship between occupational exposure and musculoskeletal disorders and symptoms. Further research is needed in order to clarify the roles of these factors and how much each contributes to the development of musculoskeletal symptoms and disorders.

\section{CONCLUSION/RECOMMENDATIONS}

\section{Need for More Useful Exposure Data}

It is clear that there is a need for exposure data that is objective, not subject to recall bias, and based upon observation. The majority of the studies used self-report as the measure of job exposures, however this may not be a valid method of ascertaining exposure for certain MSDs. Although some self-reported exposures may be useful in establishing causality, ultimately more specific exposure may be necessary to determine the required changes in order to reduce overall risk.

\section{Research on Lower Legs \& Ankle/Feet}

Based upon this review, the body of literature related to the lower legs and especially of the ankles and feet, is sparse. Most of the literature has focused on musculoskeletal symptoms and disorders of the hip and knee. Often, studies have combined the ankle/feet and lower leg symptoms with all lower extremity complaints. This method of analysis may mask significant associations specific to the ankle, feet, and lower legs.

The lack of epidemiologic studies on the lower legs and feet/ankle is in contrast to the clinical and laboratory studies of standing and leg swelling, discomfort, fatigue, and some disorders $(25,26,66)$. Also Krause (67) identified a significant association between standing and carotid atherosclerosis, which may give further insight in how workplace standing affects lower extremities as well. Future research should not only include important cofactors such as type of flooring and shoes, but also on the effect of occupational physical activities on the lower legs (67). Other areas of study that may help determine the risk factors for the development of lower extremity musculoskeletal disorders include the role of foot alignment and distribution of pressure (both on a hard surface and within the shoe) (68). The biomechanics of standing and gait may also influence lower extremity disorders and interact with workers who spend a great amount of time standing and walking. 


\section{ACKNOWLEDGMENTS}

Support for this research is provided by the National Institute on Disability and Rehabilitation Research of the United States Department of Education, Grant \#H133E980007, "Rehabilitation Engineering Research Center." The opinions contained in this publication are those of the grantee and do not necessarily reflect those of the United States Department of Education.

\section{REFERENCES}

1. Redfern MS, Chaffin DB. The effects of floor types on standing tolerance industry. In: Aghazadeh F, ed. Trends in ergonomics/human factors V. New York: Elsevier Science, 1988.

2. Work-Related Injury Query System (in conjunction with the National Electronic Injury Surveillance System), 2004.

3. Baker P, Reading I, Cooper C, Coggon D. Knee disorders in the general population and their relation to occupation. Occup Environ Med 2003; 60: 794-797.

4. O'Reilly SC, Muir KR, Doherty M. Occupation and knee pain: A community study. Osteoarthritis Cartilage 2000; 8: 78-81.

5. Maetzel A, Makela M, Hawker G, Bombardier C. Osteoarthritis of the hip and knee and mechanical occupational exposure-A systematic overview of the evidence. J Rheumatol 1997; 24: 1599-1607.

6. Lievense A, Bierma-Zeinstra A, Verhagen A, Verhaar J, Koes B. Influence of work on the development of osteoarthritis of the hip: A systematic review. J Rheumatol 2001; 28(11): 2520-2528.

7. Schouten JSAG, de Bie RA, Swaen G. An update on the relationship between occupational factors and osteoarthritis of the hip and knee. Curr Opin Rheumatol 2002; 14: 89-92.

8. Kirkeskov JL, Eenberg W. Occupation as a risk factor for knee disorders. Scand J Work Environ Health 1996; 22: $165-175$.

9. Callam MJ. Epidemiology of varicose veins. Brit J Surg 1994; 81: 167-173.

10. Evans CJ, Fowkes FGR, Hajivassiliou CA, Harper DR, Ruckley CV. Epidemiology of varicose veins: A review. Int Angiol 1994; 13(3): 263-270.

11. Hobson J. Venous insufficiency at work. Angiology 1997; 48(7): 577-582.

12. Jawien A. The influence of environmental factors in Chronic Venous Insufficiency. Angiology 2003; 54 (s1): s19-s31.

13. Kristensen TS. Cardiovascular diseases and the work environment. Scand J Work Environ Health 1989; 15: $165-179$.

14. Riddle DL, Pulisic M, Pidcoe P, Johnson RE. Risk factors for plantar-fasciitis: A matched case-control study. J Bone Joint Surg 2003; 85a(5): 872-877.

15. Ryan GA. The prevalence of musculo-skeletal symptoms in supermarket workers. Ergonomics 1989; 32(4): 359-371.

16. deZwart BCH, Broersen JPJ, Frings-Dresen MHW, van Dijk FJH. Repeated survey on changes in musculoskeletal complaints relative to age and work demands. Occup Environ Med 1997; 54: 793-799.

17. de Zwart BC, Broersen JP, van der Beek AJ, Frings-Dresen MH. Occupational classification according to work demands: An evaluation study. Int J Occup Med Environ 1997; 10(3): 283-295.

18. Merlino LA, Rosecrance JC, Anton D, Cook TM. Symptoms of musculoskeletal disorders among apprentice construction workers. Appl Occup Environ Hyg 2003; 18(1): 57-64.

19. Dawson J, Thorogood M, Marks S, Juszczak E, Dodd C, Lavis G, Fitzpatrick R. The prevalence of foot problems in older women: A cause for concern. J Public Health Med 2002; 24(2): 77-84.

20. Bergenudd H, Lindgarde F, Nilsson B. Prevalence and coincidence of degenerative changes of the hands and feet in middle age and their relationship to occupational work load, intelligence, and social background. Clin Orthop 1989; Feb (239): 306-310.

21. Akesson I, Johnsson B, Rylander L, Moritz U. Musculoskeletal disorders among female dental personnelClinical examination and a 5-year follow-up study of symptoms. Int Arch Occup Environ Health 1999; 72 : 395-403.

22. Engels JA, van der Gulden JWJ, Senden TF, van't Hof B. Work-related risk factors for musculoskeletal complaints in the nursing profession: Results of a questionnaire survey. OEM 1996; 53: 636-641.

23. Lemasters GK, Atterbury MR, Booth-Jones AD, Bhattacharya A, Ollila-Glenn N, Forrester C, Forst L. Prevalence of work related musculoskeletal disorders in active union carpenters. Occup Environ Med 1998; 55: 421-427. 
24. Gamperiene $\mathrm{M}$, Stigum $\mathrm{H}$. Work related risk factors for musculoskeletal complaints in the spinning industry in Lithuania. Occup Environ Med 1999; 56: 411-416.

25. Cham R, Redfern MS. Effect of flooring on standing comfort and fatigue. Hum Factors 2001; 43(3): 381-391.

26. Redfern MS. Influence of flooring on standing fatigue. Hum Factors 1995; 37(3): 570-581.

27. Redfern MA, Cham R. The influence of flooring on standing comfort and fatigue. AIHAJ 2000; 61: 700708.

28. Brand FN, Dannenberg AL, Abbott RD, Kannel WB. The epidemiology of varicose veins: The Framingham Study. Am J Prev Med 1988; 4: 96-101.

29. Tuchsen F, Krause N, Hannerz N, Burr H, Kristensen TS. Standing at work and varicose veins. Scand J Work Environ Health 2000; 26(5): 414-420.

30. Scott TE, LaMorte WW, Gorin DR, Menzoian JO. Risk factors for chronic venous insufficiency: A dual case-control study. J Vasc Surg 1995; 22: 622-628.

31. Kontosic I, Vukelic M, Drescik I, Mesaros-Kanjski E, Materljan E, Jonjic A. Work conditions as risk factors for varicose veins of the lower extremities in certain professions of the working population of Rijeka. Acta Med Okayama 2000; 54(1): 33-38.

32. Fowkes FGR, Lee AJ, Evans CJ, Allan PL, Bradbury AW, Ruckley CV. Lifestyle risk factors for lower limb venous reflux in the general population: Edinburgh Vein Study. Int J Epidemiol 2001; 30: 846-852.

33. Lee AJ, Evans CJ, Allan PL, Ruckley CV, Fowkes FGR. Lifestyle factors and the risk of varicose veins: Edinburgh vein study. J Clin Epidemiol 2003; 56: 171-179.

34. Lacroix P, Aboyans V, Preux PM, Houles MB, Laskar M. Epidemiology of venous insufficiency in an occupational population. Int Angiol 2003; 22: 172-176.

35. Engels JA, van der Gulden JWJ, Senden TF, Hertog CAWM, Kolk JJ, Binkhorst RA. Physical work load and its assessment among the nursing staff in nursing homes. JOM 1994; 36(3): 338-345.

36. Madelein PP, Voigt M, Arendt-Nielsen L. Subjective, physiological and biomechanical responses to prolonged manual work performed standing on hard and soft surfaces. Eur J Appl Physiol 1998; 77: 1-9.

37. Van Dieen JP, Vrielink HHEO. Evaluation of work-rest schedules with respect to the effects of postural workload in standing work. Ergonomics 1998; 41(12): 1832-1844.

38. Jensen LK, Eenberg W. Occupation as a risk factor for knee disorders. Scand J Work Environ Health 1996; 22: $165-175$.

39. Lau EC, Cooper C, Lam D, Chan VNH, Tsang KK, Sham A. Factors associated with osteoarthritis of the hip and knee in Hong Kong Chinese: Obesity, joint injury and occupational activities. Am J Epidemiol 2000; 152: 855-862.

40. Yoshimura N, Nishioka S, Kinoshita H, Hori N, Nishioka T, Ryujin M, Mantani Y, Miyake M, Coggon D, Cooper C. Risk factors for knee osteoarthritis in Japanese women: Heavy weight, previous joint injuries, and occupational activities. J Rheumatol 2004; 31: 157-162.

41. Cooper C, McAlindon T, Coggon D, Egger P, Dieppe P. Occupational activity and osteoarthritis of the knee. Ann Rheum Dis 1994; 53: 90-93.

42. Coggon D, Croft P, Kellingray S, Barrett D, McLaren M, Cooper C. Occupational physical activities and osteoarthritis of the knee. Arthritis Rheum 2000; 43(7): 1443-1449.

43. Bagge E, Bjelle A, Eden S, Svanborg A. Factors associated with radiographic osteoarthritis: Results from the population study of 70-year-old people in Goteberg. J Rheumatol 1991; 18: 1218-1222.

44. Felson DT, Hannan MT, Naimark A, Berkeley J, Gordon G, Wilson PWF, Anderson J. Occupational physical demands, knee bending, and knee osteoarthritis: Results from the Framingham study. J Rheumatol 1991; 18: 1587-1592.

45. Dawson J, Juszczak E, Thorogood M, Marks S-A, Dodd C, Fitzpatrick R. An investigation of risk factors for symptomatic osteoarthritis of the knee in women using a life course approach. J Epidemiol Community Health 2003; 57: 823-830.

46. Manninen P, Heliovaara M, Riihimaki H, Suoma-Iainen O. Physical workload and the risk of severe knee osteoarthritis. Scand J Work Environ Health 2002; 28(1): 25-32.

47. Teichtahl A, Wluka A, Cicuttini FM. Abnormal biomechanics: A precursor or result of knee osteoarthritis? Br J Sports Med 2003; 37: 289-290.

48. Kerrigan DC, Todd MK, Riley PO. Knee osteoarthritis and high-heeled shoes. Lancet 1998; 351: 13991401 .

49. Baker P, Coggon D, Reading I, Barrett D, McLaren M, Cooper C. Sports injury, occupational physical activity, joint laxity and meniscal damage. J Rheumatol 2002; 29: 557-563.

50. Chen JC, Denneriein JT, Shih TS, Chen CJ, Cheng YM, Chang WP, Ryan LM, Christiani, DC. Knee pain and driving duration: A secondary analysis of the taxi drivers' health study. Am J Public Health 2004; 94(4): 575-581.

51. Miranda H, Vikari-Juntura E, Martikainen R, Riihimaki H. A prospective study on knee pain and its risk factors. Osteoarthritis Cartilage 2002; 10: 623-630.

52. Jensen LK, Mikkelsen S, Loft IP, Eenberg W. Work-related knee disorders in floor layers and carpenters. JOEM 2000; 42(8): 835-842. 
53. Lagerstrom M, Wenemark M, Hagberg M, Hjelm EW. Occupational and individual factors related to musculoskeletal symptoms in five body regions among Swedish nursing personnel. Int Arch Occup Environ Health 1995; 68: 27-35.

54. Sobti A, Cooper C, Inskip H, Searle S, Coggon D. Occupational physical activity and long-term risk of musculoskeletal symptoms: A national survey of post office pensioners. Am J Ind Med 1997; 32: 76-83.

55. Tuchsen F, Hannerz H, Jensen MV, Krause N. Socioeconomic status, occupation and risk of hospitalization due to coxarthrosis in Denmark 1981-99. Ann Rheum Dis 2003; 62: 1100-1105.

56. Croft P, Cooper C, Wickham C, Coggon D. Osteoarthritis of the hip and occupational activity. Scand $J$ Work Environ Health 1992; 18: 59-63.

57. Roach KE, Persky V, Miles T, Budiman-Mak E. Biomechanical aspects of occupation and osteoarthritis of the hip: A case-control study. J Rheumatol 1994; 21: 2334-2340.

58. Pope DP, Hunt IM, Birrell FN, Silman AJ, Macfarlane GJ. Hip pain onset in relation to cumulative workplace and leisure time mechanical load: A population based case-control study. Ann Rheum Dis 2003; 62: 322-326.

59. Tuchsen F, Hannerz H, Burr H, Lund T, Krause N. Risk factors predicting hip pain in a 5-year prospective cohort study. Scand J Work Environ Health 2003; 29(1): 35-39.

60. Toren A, Oberg K, Lembke B, Enlund K, Rask-Andersen A. Tractor-driving hours and their relation to self-reported low-back and hip symptoms. Appl Ergon 2002; 33: 139-146.

61. Wiktorin C, Hjelm EW, Winkel J, Koster M, Stockholm MUSIC I Study Group. Reproducibility of a questionnaire for assessment of physical load during work and leisure time. JOEM 1996; 38(2): 190-201.

62. Wiktorin C, Karlqvist L, Winkel J, Stockholm MUSIC I study group. Scand J Work Environ Health 1993; 19: 208-214.

63. Keyserling WM, Brouwer M, Silverstein BA. A checklist for evaluating ergonomic risk factors resulting from awkward posture of the legs, trunk and neck. Int J Ind Ergon 1992; 9: 283-301.

64. Kivimaki J. Occupationally related ultrasonic findings in carpet and floor layers' knees. Scand J Work Environ Health 1992; 34(5): 496-499.

65. Myllymaki T, Tikkakoski T, Typpo T, Kivimaki J, Suramo I. Carpet-layer's knee: An ultrasonographic study. Acta Radiol 1993; 18: 400-402.

66. Madeleine P, Voigt M, Arendt-Nielsen L. Subjective, physiological and biomechanical responses to prolonged manual work performed standing on hard and soft surfaces. Eur J Appl Physiol 1998; 77: 1-79.

67. Krause N, Kaplan GA, Cohen RD, Salonen R, Salonen JT. Standing at work and progression of carotid atherosclerosis. Scand J Work Environ 2000; 26(3): 227-236.

68. Krijnen RM, de Boer EM, Ader HJ, Bruynzeel DP. Venous insufficiency in male workers with a standing profession. Part 1: Epidemiology. Dermatology 1997; 194(2): 111-120. 